Check for updates

Cite this: RSC Adv., 2021, 11, 30415

\title{
Synthesis of spirocyclic $\Delta^{4}$-isoxazolines via [3+2] cycloaddition of indanone-derived ketonitrones with alkynes $\dagger$
}

\author{
Yilin Liu, (D) *ab Jiaxue Liu, ${ }^{a}$ Yan-Yun Liu, (D) *a Boxiao Tang, ${ }^{a}$ Hongwei Lin, ${ }^{a}$ \\ Yuanxiang $\mathrm{Li}^{\mathrm{a}}$ and Lin Zhang ${ }^{\mathrm{a}}$
}

A [3 + 2] cycloaddition of indanone-derived nitrones and alkynes under mild conditions is developed, allowing facile synthesis of spirocyclicindenyl isoxazolines with structural diversity. The sequential protocol of generated in situ ketonitrone from unsaturated ketones and $\mathrm{N}$-alkylhydroxylamines is also achieved successfully, affording the desired products in considerable yield with moderate to good diastereoselectivity. Moreover, the spirocyclic product can be conveniently transformed into indenylbased allylic alcohol and enamide.

Received 11th August 2021

Accepted 5th September 2021

DOI: 10.1039/d1ra06063e

rsc.li/rsc-advances

and expensive reagents, ${ }^{4,5 c, 6 c}$ or multistep manipulations as well as uneconomical atomic transformations..$^{5,6 a}$ Consequently, the development of an environmentally friendly and atomeconomical cycloaddition of novel nitrone generated in situ for the synthesis of highly functionalized isoxazoline is still of great interest.

Spiroisoxazolines have been received intensive attention because the incorporation of a rigid spiro-ring can reduce the conformational entropy penalty upon binding with a protein target in modern drug discovery. ${ }^{9}$ However, the application of cycloaddition reaction of nitrone to construct spiroisoxazolines

complicated operation and the separation of unstable nitrones. Despite great progress being made in the cycloaddition of aldonitrones with cyclopropanes, ${ }^{4}$ olefins, ${ }^{5}$ and alkynes, ${ }^{6}$ however, the cycloaddition of ketonitrones generated in situ for preparation of 4-isoxazoline remains scarce. In 2009, Frontier and coworkers reported that the $[3+2]$ dipolar cycloaddition of an electron-deficient alkyne and a ketonitrone generated in situ from condensation of acetone and $N$-methylhydroxylamine gives an isolable isoxazoline in $79 \%$ yield (Scheme 1a). ${ }^{7}$ Recently, Woo developed an efficient visible-light photoredoxcatalyzed $[3+2]$ cycloaddition of oxaziridines with alkynes to give 4-isoxazoline in high yield, this novel strategy involves in situ generation of ketonitrones from oxaziridines through a SET way (Scheme 1b). ${ }^{8}$ However, most of these powerful approaches suffer from the use of transition metal catalysts and unstable

${ }^{a}$ Hunan Engineering Laboratory for Preparation Technology of Polyvinyl Alcohol (PVA) Fiber Material, Institute of Organic Synthesis, Huaihua University, Huaihua 41800o, China.E-mail: liuyilinhn@126.com; liuyanyun314@sina.com

${ }^{b}$ CAS Key Laboratory of Molecular Recognition and Function, Institute of Chemistry, Chinese Academy of Sciences, Beijing 100190, China

$\dagger$ Electronic supplementary information (ESI) available: Experimental procedures, X-ray crystal structures of compounds 6 and 7. CCDC 2068718 and 2068719. Copies of NMR spectra. For ESI and crystallographic data in CIF or other electronic format see DOI: 10.1039/d1ra06063e

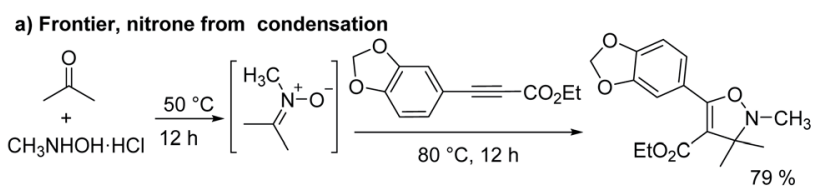

b) Woo, nitrone from the oxaziridines by SET

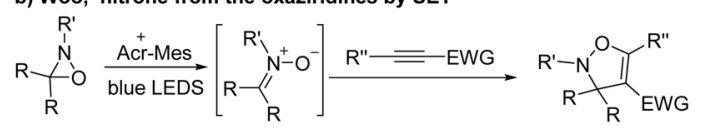

c) our previous work, cycloaddition of indanone-derived nitrone with Alkyne
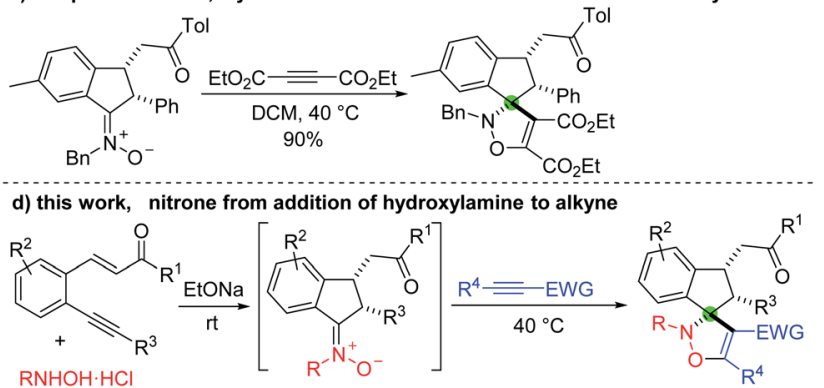

Scheme 1 Cycloadditions of ketonitrone generated in situ with alkynes. 
is largely undeveloped, because methods for the synthesis of cyclic ketonitrone are still not rich and mostly limited to specific substrates scope such as cyclic ketone derived nitrones, ${ }^{\mathbf{1 0}}$ isatin ketonitrones, ${ }^{\mathbf{1 1}}$ sugar ketonitrones, ${ }^{\mathbf{1 2}}$ fluorenone nitrones. ${ }^{\mathbf{1 3}}$ Therefore, only a few examples using these compounds as substrates to synthesize spirocyclic compounds have been reported. Oxyallyl cations, ${ }^{\mathbf{1 4}}$ cyclopropanes, ${ }^{11 f}$ aza-oxyallyl cations, ${ }^{15}$ olefins ${ }^{\mathbf{1 1 a}, \boldsymbol{b}, \boldsymbol{d}, \mathbf{1 2 , 1 6}}$ gave the expected cycloadducts in high yields, while reactions with acetylenes seldom gave the corresponding spiroisoxazolines. Instead, non spiro products arising from transformations of the initially formed spiroisoxazoline are produced in these cases. ${ }^{\mathbf{1 3 c , 1 7}}$ For example, in 2012, Anderson and co-workers reported that the reaction of alkynes with $N$-vinyl fluorenone nitrones provides the fluorene-tethered isoxazoles at high temperature via a cyclization and elimination process. $^{13 c}$ In contrast, Prathapan and co-workers discovered that the cycloadduct resulting from the reaction of $N$-phenyl fluorenone nitrones and electron deficient acetylenes was formed predominantly initially and then could undergo rearrangement easily to give $3(2 \mathrm{H})$-furanone at room temperature. ${ }^{17 b}$ Therefore, the continuous development of novel cyclic ketonitrones for the discovery of spirocyclic drug candidates is highly desirable.

Recently, we developed a carbonyl-directed addition of $\mathrm{N}$ alkylhydroxylamines to unactivated alkynes with high stereoselectivity. This strategy enables the facile synthesis of indanone-derived nitrones, which was subjected successfully to $[3+2]$ cycloadditions with diethyl acetylenedicarboxylate (DEAD) in dichloromethane (DCM) to give spiro-isoxazoline in 90\% yield (Scheme 1c). ${ }^{20}$ This privilege skeleton prompted us to further expand the substrate scope. Herein, we wish to report our efforts on the $[3+2]$ cycloaddition of indanone ketonitrones with alkynes under mild conditions (Scheme 1d).

\section{Results and discussion}

It was found that the amount of DEAD $\mathbf{4 a}$ in cycloadditions could be decreased to 1.5 equivalent, and the same reactivity was observed. The relative configuration of product spiroindenyl isoxazoline $\mathbf{5}$ was assigned by its analogue X-ray diffraction analysis reported by $\mathrm{us}^{20}$ As shown in Table 1 , a variety of $N$-benzyl indanone-derived nitrones underwent cycloadditions with DEAD 4a in DCM at $40{ }^{\circ} \mathrm{C}$ smoothly, affording the corresponding spiroindenyl isoxazolines in good to excellent yields, albeit with low dr values (5aaa-qaa). It was interesting to find that when the $\mathrm{R}^{1}$ group was methyl substituent, the nitrone 3 ga produced the desired products in higher dr value, compared to nitrones with aryl group at position $\mathrm{R}^{1}$ (5aaa-faa $v s$. 5gaa). Spiroindenyl isoxazolines could be obtained in excellent yields when indanone-derived nitrones 3ha-ma were used as substrates. The reaction tolerated different groups at position $\mathrm{R}^{2}$ in nitrones 3 , affording the desired products in good to excellent yields (5naa-qaa). It is worth noting that naphthalenyl isoxazoline 5qaa was furnished in $87 \%$ yield with a high $21: 1 \mathrm{dr}$, which may attribute to the steric hindrance effect. To our pleasure, $N$-cyclohexenyl indanone-derived nitrone $\mathbf{3 d \mathbf { d }}$ was also an effective substrate for this reaction to
Table 1 Scope of indanone-derived nitrone ${ }^{a}$
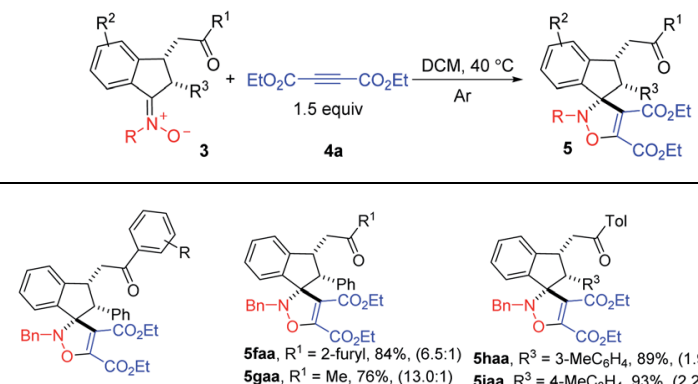

5aaa, R $=$ H, 99\%, (1.8:1)

5faa, $R^{1}=2$-furyl, 84\%, (6.5:1) 5haa, $\mathrm{R}^{3}=3-\mathrm{MeC}_{6} \mathrm{H}_{4}, 89 \%$, (1.9:1) baa, $R=2-M e, 76 \%,(1.1: 1)$ Scaa, $R=3$-Me, $95 \%,(1.8: 1)$ 5eaa, $R=4-\mathrm{Br}, 92 \%,(1.4: 1)$

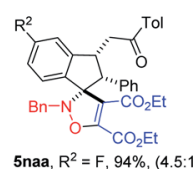

5oaa, $R^{2}=C l, 90 \%,(2.5: 1)$

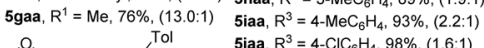

5jaa, $\mathrm{R}^{3}=4-\mathrm{ClC}_{6} \mathrm{H}_{4}, 98 \%,(1.6: 1)$ 5kaa, $\mathrm{R}^{3}=4-\mathrm{BrC}_{6} \mathrm{H}_{4}, 91 \%,(1.1: 1)$ 5laa, $\mathrm{R}^{3}=4-\mathrm{MeOC}_{6} \mathrm{H}_{4}, 99 \%,(6.5: 1)$ 5maa, $R^{3}=1$-cyclohexenyl, $92 \%,(6.8: 1)$
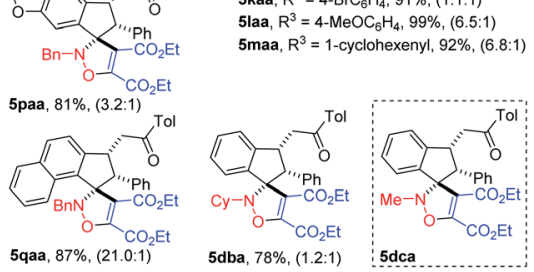

${ }^{a}$ All reactions were carried out with 3 ( $\left.0.20 \mathrm{mmol}\right), \mathbf{4 a}(1.5$ equiv.), and DCM $(3.0 \mathrm{~mL}), 17-21 \mathrm{~h}$ unless otherwise stated; isolated yield based on 3 ; the dr ratio is given in brackets and determined by ${ }^{1} \mathrm{H}$ NMR analysis (see ESI for details).

furnish the desired product 5dba in good yield. However, $N$ methyl isoxazoline 5dca failed to be furnished, because nitrone 3dc is too unstable to be separated.

Next, to further probe the generality of this cycloaddition reaction, a variety of alkynes $\mathbf{4 b} \mathbf{b}$ j were treated with indanonederived nitrone 3da. The results are shown in Table 2 (5dabdaj). Although dimethyl acetylenedicarboxylate, ethyl propionate, and 3-butyn-2-one gave the corresponding spiroindenyl isoxazoline in high yields in dichloromethane, the reaction of

Table 2 Scope of alkynes ${ }^{a}$
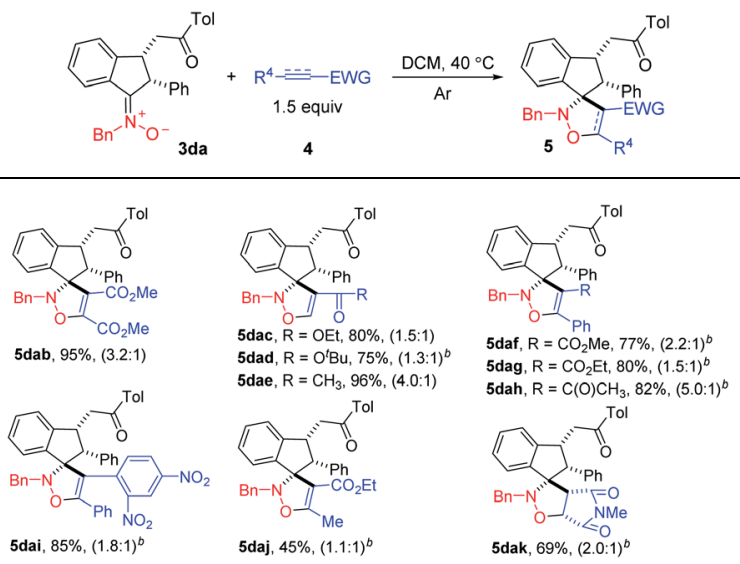

${ }^{a}$ All reactions were carried out with $3 \mathrm{da}(0.20 \mathrm{mmol}), 4$ (1.5 equiv.), and DCM $(3.0 \mathrm{~mL}), 17-21 \mathrm{~h}$ unless otherwise stated; isolated yield based on 3da; the dr ratio is given in brackets and determined by ${ }^{1} \mathrm{H}$ NMR analysis (see ESI for details). ${ }^{b}$ The reaction was carried out in $\mathrm{CHCl}_{3}$ $(3.0 \mathrm{~mL})$ at $80^{\circ} \mathrm{C}$. 
tert-butyl propionate, methyl phenylpropiolate, ethyl phenylpropiolate, 4-phenyl-3-butyn-2-one, and ethyl 2-butynoate needed to be carried out in chloroform at higher temperature to obtain satisfactory yields (5dab, 5dac, 5dae $v s$. 5dad, 5daf-dah, 5daj). To our delight, cycloaddition reaction of nitrone 3da with diphenylethyne also went smoothly to give diphenyl-4isoxazoline (5dai), which may be a potential inhibitor of cyclooxygenase-2 with analgesic and antiinflammatory activity according to the study reported by Knaus. ${ }^{1 a}$ It was found that electron deficient olefin was also a good partner in cycloaddition reaction with indanone-derived nitrone, as $N$-methylmaleimide could afford the spiroindenyl isoxazolidine in moderate yield (5dak).

DCM was used as the solvent in both the preparation of indanone-derived nitrone and the cycloaddition reaction of nitrone with electron deficient alkyne, therefore, we envisioned that cycloaddition of nitrones generated in situ from unsaturated ketone $\mathbf{1}$, and $\mathrm{N}$-alkylhydroxylamine 2, with alkyne $\mathbf{4}$ for synthesis of spiroindenyl isoxazoline was possible. Indeed, the cycloaddition of nitrones generated in situ went smoothly to afford the spiroindenyl isoxazoline in good yield, and the results are summarized in Table 3. Of note is that 5dca can be afforded successfully in a yield of $45 \%$. Surprisingly, this cycloaddition gave higher dr value than cycloaddition of pre-prepared nitrone in Table 1. The mechanism is still not clear currently, according to the previous literature ${ }^{17-19}$ and experimental results, the reaction process may be determined by the attack of nucleophilic oxygen anion in nitrone moiety on the carbon-carbon triple bond in alkynes, ${ }^{19}$ and the reason is probably that this cycloaddition, at least in part, follows a two-step mechanism, while cycloaddition of pre-prepared nitrone in Table 1 proceeds in a concerted manner.

Table $3[3+2]$ cycloaddition reaction of generated in situ ketonitrone ${ }^{a}$
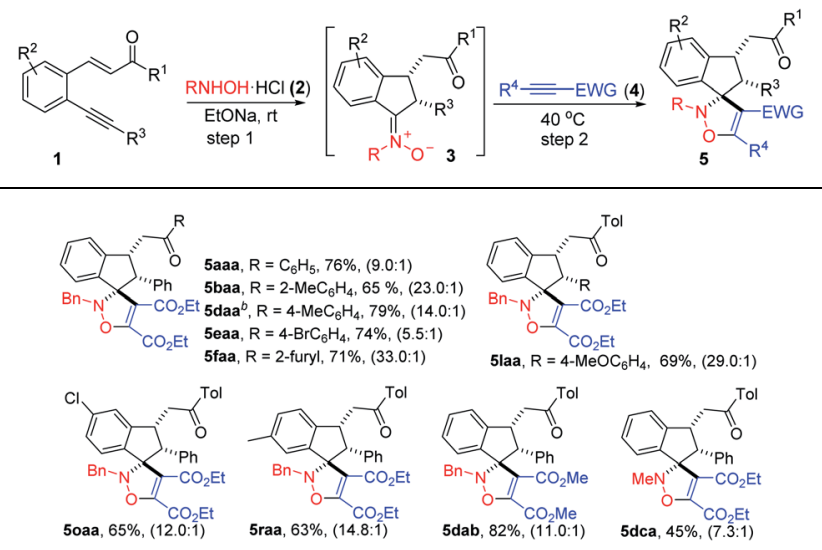

\footnotetext{
${ }^{a}$ All reactions were carried out with $1(0.50 \mathrm{mmol}), 2(0.50 \mathrm{mmol})$, EtONa (1.3 equiv.), and DCM $(5.0 \mathrm{~mL}), 12-24 \mathrm{~h}$ for step one, then 4 (1.5 equiv.) was added, $17-21 \mathrm{~h}$ for step two unless otherwise stated; isolated yield based on $\mathbf{1}$; the $\mathrm{dr}$ ratio is given in brackets and determined by ${ }^{1} \mathrm{H}$ NMR analysis (see ESI for details). ${ }^{b}$ The reactions were carried out with $4 \mathrm{mmol}$ scale of $\mathbf{1 d}$.
}

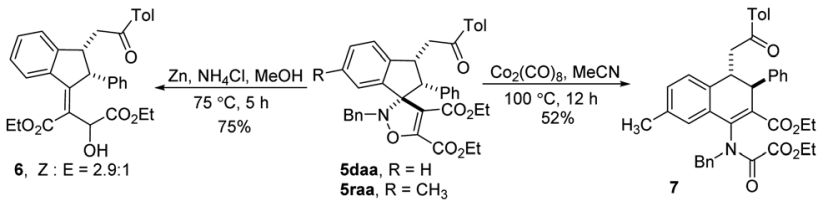

Scheme 2 Transformations of spiroisoxazolines.

With the novel spiroisoxazolines in hand, subsequently, transformations of isoxazoline were investigated (Scheme 2). 4Isoxazoline 5daa underwent reductive cleavage of the $\mathrm{N}-\mathrm{O}$ bond successfully in the present of zinc powder and $\mathrm{NH}_{4} \mathrm{Cl}$ at $75^{\circ} \mathrm{C}$, affording allylic alcohol 6 in a yield of $75 \%{ }^{8,21}$ Besides, it was found that $\mathrm{Co}_{2}(\mathrm{CO})_{8}$ catalyzed rearrangement of 4 -isoxazoline 5raa could occur in MeCN, giving enamide 7 in $52 \%$ yield, instead of ring contraction product acylaziridines. ${ }^{22}$ While the mechanism for $\mathrm{Co}_{2}(\mathrm{CO})_{8}$ catalyzed rearrangement is not clear at present, further examination of the rearrangement reaction conditions and mechanism will be carried out in due course.

\section{Conclusions}

In summary, we have reported a novel [3+2] cycloaddition between indanone-derived nitrones and electron deficient alkynes to give a series of spiroindenyl isoxazolines under mild conditions in moderate to good yields. To the best of our knowledge, this is the first example of [3+2] cycloaddition of indanone-derived nitrones generated in situ, giving the corresponding spiroindenyl isoxazolines in high diastereoselectivity. Application of these spiroindenyl isoxazolines and expansion of the scope of dipolarophiles for synthesis of other novel spiroindenyl compounds are currently under investigation in our laboratory.

\section{Experimental}

\section{General information}

All ${ }^{1} \mathrm{H},{ }^{13} \mathrm{C}$, and ${ }^{19} \mathrm{~F}$ NMR spectra were recorded at ambient temperatures on a Bruker $400 \mathrm{MHz}$ or $500 \mathrm{MHz}$ advance spectrometer with tetramethylsilane as internal standard. Highresolution mass spectra (HRMS) were recorded on an Agilent 1290 or GCT Premier Mass Spectrometer using ESI-TOF or EI (electrospray ionization time of flight). All reactions were monitored by thin-layer chromatography. Column chromatography (petroleum ether/ethyl acetate) was performed on silica gel (200-300 mesh). 2,4-Dinitro-1-(phenylethynyl)benzene $\mathbf{4 i}^{\mathbf{2 3}}$ was prepared according to literature procedure, and other reagents were purchased from commercial suppliers and used without further purification.

Representative procedure for the synthesis of spiroindenyl isoxazoline 5 (Table 1, 5aaa). To a dried Schlenk flask was charged with 3aa $(0.0863 \mathrm{~g}, 0.20 \mathrm{mmol})$, diethyl acetylenedicarboxylate $(0.0511 \mathrm{~g}, 0.30 \mathrm{mmol})$, and DCM $(3.0 \mathrm{~mL})$ under argon. The reaction mixture was stirred at $40{ }^{\circ} \mathrm{C}$ for $20 \mathrm{~h}$, and then was concentrated. The crude residue was purified by flash chromatography on silica gel (petroleum ether/ethyl acetate $=$ 
$10: 1, \mathrm{v} / \mathrm{v})$ to afford the desired product 5aaa as a light yellow solid (0.1191 g, 99\% yield, $1.8: 1 \mathrm{dr})$.

Diethyl 2'-benzyl-3-(2-oxo-2-phenylethyl)-2-phenyl-2,3-dihydro- $2^{\prime} H$-spiro[indene-1, $3^{\prime}$-isoxazole]- $4^{\prime}, 5^{\prime}$-dicarboxylate (5aaa), $1.8: 1 \mathrm{dr}$. Purified by silica gel column chromatography $(10: 1$ petroleum ether/ethyl acetate): $119.1 \mathrm{mg}$, 99\% yield; light yellow solid, mp 120-122 ${ }^{\circ} \mathrm{C}$, IR (film) 1739, 1709, 1474, 1303, 1231, $1188 \mathrm{~cm}^{-1}$; ${ }^{1} \mathrm{H}$ NMR (500 MHz, $\left.\mathrm{CDCl}_{3}\right) \delta 7.93(\mathrm{~d}, J=10.0 \mathrm{~Hz}$, $0.7 \mathrm{H}), 7.79(\mathrm{~d}, J=10.0 \mathrm{~Hz}, 1.3 \mathrm{H}), 7.67(\mathrm{t}, J=5.0 \mathrm{~Hz}, 1.3 \mathrm{H}), 7.57-$ $7.33(\mathrm{~m}, 5.7 \mathrm{H}), 7.32-7.11(\mathrm{~m}, 10 \mathrm{H}), 4.50(\mathrm{t}, J=10.0 \mathrm{~Hz}, 0.3 \mathrm{H})$, 4.25-4.07 (m, 5.3H), $4.02(\mathrm{~d}, J=15.0 \mathrm{~Hz}, 0.7 \mathrm{H}), 3.87(\mathrm{dd}, J=$ 15.0, $5.0 \mathrm{~Hz}, 0.7 \mathrm{H}), 3.57-3.43(\mathrm{~m}, 2.3 \mathrm{H}), 3.36(\mathrm{dd}, J=20.0$, $10.0 \mathrm{~Hz}, 0.4 \mathrm{H}), 3.22(\mathrm{dd}, J=15.0,5.0 \mathrm{~Hz}, 0.3 \mathrm{H}), 1.29-1.20(\mathrm{~m}$, $4.1 \mathrm{H}), 1.13$ (d, $J=10.0 \mathrm{~Hz}, 1.9 \mathrm{H}) ;{ }^{13} \mathrm{C} \mathrm{NMR}\left(125 \mathrm{MHz}, \mathrm{CDCl}_{3}\right.$ ) $\delta$ 199.5, 199.1, 162.7, 162.4, 159.2, 155.4, 154.8, 148.0, 147.0, $139.8,137.8,137.5,137.4,137.4,137.2,137.0,135.5,133.6$, $133.4,133.1,131.6,130.5,130.0,129.7,129.1,129.0,128.8$, $128.7,128.6$, 128.6, 128.4, 128.3, 128.2, 128.1, 128.0, 127.6, $127.6,127.4,127.2,127.2$, 127.1, 126.6, 126.3, 126.0, 124.7, 113.1, 108.7, 100.2, 85.3, 84.0, 62.6, 62.6, 61.4, 60.9, 60.8, 60.6, 60.2 , 58.4, 43.9, 42.6, 42.3, 40.4, 14.2,14.1, 14.0, 14.0; HRMS(ESI) calcd for $\mathrm{C}_{38} \mathrm{H}_{36} \mathrm{NO}_{6}[\mathrm{M}+\mathrm{H}]^{+}$602.2537, found 602.2543.

Diethyl 2'-benzyl-3-(2-oxo-2-(o-tolyl)ethyl)-2-phenyl-2,3-dihydro- $2^{\prime} H$-spiro[indene-1,3'-isoxazole $]-4^{\prime}, 5^{\prime}$-dicarboxylate (5baa), $1.1: 1 \mathrm{dr}$. Purified by silica gel column chromatography $(20: 1$ petroleum ether/ethyl acetate): $93.6 \mathrm{mg}$, 76\% yield; light yellow solid, mp 87-89 ${ }^{\circ} \mathrm{C}$, IR (film) 1747, 1712, 1456, 1371, 1299, $1187 \mathrm{~cm}^{-1} ;{ }^{1} \mathrm{H}$ NMR (400 MHz, $\left.\mathrm{CDCl}_{3}\right) \delta 7.64-7.62(\mathrm{~m}, 1 \mathrm{H}), 7.58$ $(\mathrm{d}, J=8.0 \mathrm{~Hz}, 0.5 \mathrm{H}), 7.45-7.43(\mathrm{~m}, 2 \mathrm{H}), 7.39-7.36(\mathrm{~m}, 1.5 \mathrm{H})$, 7.34-7.26 (m, 4H), 7.25-7.10 (m, 9H), 4.49-4.43 (m, 0.6H), 4.21$4.12(\mathrm{~m}, 4.8 \mathrm{H}), 4.00(\mathrm{~d}, J=12.0 \mathrm{~Hz}, 0.5 \mathrm{H}), 3.86(\mathrm{~d}, J=16.0 \mathrm{~Hz}$, $0.5 \mathrm{H}), 3.82(\mathrm{~d}, J=12.0 \mathrm{~Hz}, 0.5 \mathrm{H}), 3.52(\mathrm{~d}, J=12.0 \mathrm{~Hz}, 0.5 \mathrm{H})$, $3.50-3.42(\mathrm{~m}, 1.3 \mathrm{H}), 3.32-3.25(\mathrm{~m}, 0.6 \mathrm{H}), 3.16$ (dd, $J=16.0$, $4.0 \mathrm{~Hz}, 0.5 \mathrm{H}), 2.48(\mathrm{~s}, 1.6 \mathrm{H}), 2.36(\mathrm{~s}, 1.4 \mathrm{H}), 1.29-1.18(\mathrm{~m}, 4.6 \mathrm{H})$, $1.13(\mathrm{~d}, J=8.0 \mathrm{~Hz}, 1.4 \mathrm{H}) ;{ }^{13} \mathrm{C} \mathrm{NMR}\left(100 \mathrm{MHz}, \mathrm{CDCl}_{3}\right) \delta 203.3$, 202.8, 162.7, 162.4, 159.3, 159.2, 155.5, 154.8, 148.2, 147.0, $138.7,138.4,138.3,137.8,137.6,137.5,137.3$, 137.2, 137.0, $135.5,132.3,132.1,131.7,131.7,131.4,130.5,130.0,129.7$, $129.1,129.0,128.7,128.6$, 128.5, 128.3, 128.2, 128.0, 127.6, $127.4,127.2,127.2,127.1,126.5,126.4,126.1,125.9,125.7$, $124.5,108.7,127.2,85.3,84.1,62.6,61.5,60.9,60.8,60.6,60.2$, 58.4, 45.6, 45.0, 43.9, 40.7, 29.9, 21.7, 21.4, 14.2, 14.1, 14.0, 14.0; HRMS(ESI) calcd for $\mathrm{C}_{39} \mathrm{H}_{38} \mathrm{NO}_{6}[\mathrm{M}+\mathrm{H}]^{+}$616.2694, found 616.2690 .

Diethyl 2'-benzyl-3-(2-oxo-2-( $m$-tolyl)ethyl)-2-phenyl-2,3-dihydro-2 ${ }^{\prime} H$-spiro[indene-1, $3^{\prime}$-isoxazole]- $4^{\prime}, 5^{\prime}$-dicarboxylate (5caa), $1.8: 1 \mathrm{dr}$. Purified by silica gel column chromatography $(20: 1$ petroleum ether/ethyl acetate): $117.0 \mathrm{mg}$, 95\% yield; light yellow oil, IR (film) 1745, 1701, 1496, 1370, 1181, $1108 \mathrm{~cm}^{-1} ;{ }^{1} \mathrm{H}$ NMR $\left(500 \mathrm{MHz}, \mathrm{CDCl}_{3}\right) \delta 7.69(\mathrm{~d}, J=10.0 \mathrm{~Hz}, 2 \mathrm{H}), 7.59(\mathrm{~s}, 0.7 \mathrm{H}), 7.46-$ $7.40(\mathrm{~m}, 2 \mathrm{H}), 7.36-7.26(\mathrm{~m}, 7 \mathrm{H}), 7.24-7.12(\mathrm{~m}, 5 \mathrm{H}), 6.69(\mathrm{t}, J=$ $10.0 \mathrm{~Hz}, 0.5 \mathrm{H}), 6.79(\mathrm{~d}, J=5.0 \mathrm{~Hz}, 1 \mathrm{H}), 4.48(\mathrm{t}, J=10.0 \mathrm{~Hz}$, $0.6 \mathrm{H}), 4.26-4.10(\mathrm{~m}, 4.5 \mathrm{H}), 4.02(\mathrm{~d}, J=15.0 \mathrm{~Hz}, 0.4 \mathrm{H}), 3.88-3.85$ $(\mathrm{m}, 1.3 \mathrm{H}), 3.53-3.43(\mathrm{~m}, 1.7 \mathrm{H}), 3.34(\mathrm{dd}, J=20.0,10.0 \mathrm{~Hz}, 0.7 \mathrm{H})$, $3.20(\mathrm{~d}, J=15.0 \mathrm{~Hz}, 0.6 \mathrm{H}), 2.38(\mathrm{~s}, 1.9 \mathrm{H}), 2.31(\mathrm{~s}, 1.1 \mathrm{H}), 1.28-$ $1.20(\mathrm{~m}, 5 \mathrm{H}), 1.12(\mathrm{t}, J=10.0 \mathrm{~Hz}, 1.3 \mathrm{H}) ;{ }^{13} \mathrm{C} \mathrm{NMR}(125 \mathrm{MHz}$, $\left.\mathrm{CDCl}_{3}\right) \delta 200.0,199.6,162.9,162.4,159.3,156.1,155.6,154.9$,
148.0, 147.0, 138.6, 138.5, 137.5, 137.4, 137.2, 137.1, 137.0, $135.6,134.2$, 133.9, 131.6, 130.5, 130.0, 129.8, 129.7, 128.9, 128.7 , 128.6, 128.6, 128.2, 128.2, 128.0, 127.6, 127.5, 127.3, 127.2 , 127.2, 127.1, 126.6, 126.3, 126.0, 125.6, 125.4, 124.7, 120.6, 115.5, 107.3, 85.3, 84.0, 62.6, 61.4, 60.9, 60.7, 60.2, 58.4, $44.0,42.6,42.3,40.5,29.9,21.5,14.2,14.1,14.0,14.0$; HRMS(ESI) calcd for $\mathrm{C}_{39} \mathrm{H}_{38} \mathrm{NO}_{6}[\mathrm{M}+\mathrm{H}]^{+}$616.2694, found 616.2696.

Diethyl 2'-benzyl-3-(2-oxo-2-( $p$-tolyl)ethyl)-2-phenyl-2,3-dihydro-2' $H$-spiro[indene-1, $3^{\prime}$-isoxazole $]-4^{\prime}, 5^{\prime}$-dicarboxylate (5daa), 2.9 : 1 dr. Purified by silica gel column chromatography (20: 1 petroleum ether/ethyl acetate): $118.2 \mathrm{mg}, 96 \%$ yield; light yellow solid, mp 127-129 ${ }^{\circ} \mathrm{C}$, IR (film) 1743, 1709, 1474, 1303, 1231, $1181 \mathrm{~cm}^{-1}$; ${ }^{1} \mathrm{H}$ NMR (500 MHz, $\mathrm{CDCl}_{3}$ ) $\delta 7.83(\mathrm{~d}, J=10.0 \mathrm{~Hz}$, $1.5 \mathrm{H}), 7.70-7.66(\mathrm{~m}, 1 \mathrm{H}), 7.47-7.12(\mathrm{~m}, 15.5 \mathrm{H}), 4.50(\mathrm{t}, J=$ $10.0 \mathrm{~Hz}, 0.7 \mathrm{H}), 4.26-4.10(\mathrm{~m}, 4.5 \mathrm{H}), 4.02(\mathrm{~d}, J=15.0 \mathrm{~Hz}, 0.3 \mathrm{H})$, $3.87(\mathrm{t}, J=10.0 \mathrm{~Hz}, 1.5 \mathrm{H}), 3.57-3.40(\mathrm{~m}, 1.4 \mathrm{H}), 3.33(\mathrm{dd}, J=20.0$, $10.0 \mathrm{~Hz}, 0.8 \mathrm{H}), 3.18(\mathrm{~d}, J=20.0 \mathrm{~Hz}, 0.7 \mathrm{H}), 2.38(\mathrm{~s}, 2.2 \mathrm{H}), 2.36(\mathrm{~s}$, $0.8 \mathrm{H}), 1.28-1.20(\mathrm{~m}, 5.2 \mathrm{H}), 1.12(\mathrm{t}, J=10.0 \mathrm{~Hz}, 0.8 \mathrm{H}) ;{ }^{13} \mathrm{C} \mathrm{NMR}$ $\left(125 \mathrm{MHz}, \mathrm{CDCl}_{3}\right) \delta 199.1,198.8,162.8,162.5,159.2,155.4$, $154.8,148.0,147.0,144.2,143.8,137.4,137.0,135.6,135.0$, 134.6 , 131.6, 130.5, 130.0, 129.6, 129.4, 129.3, 129.0, 128.6, $128.6,128.5$, 128.3, 128.2, 128.2, 128.0, 127.6, 127.5, 127.3, 127.2, 127.1, 127.0, 126.6, 126.3, 125.9, 124.7, 108.8, 107.3, 85.3, 84.0, 62.5, 61.4, 60.8, 60.6, 60.2, 58.4, 43.9, 42.4, 42.1, 40.5, 29.9, $21.8,14.2,14.1,14.0,14.0 ; \mathrm{HRMS}(\mathrm{ESI})$ calcd for $\mathrm{C}_{39} \mathrm{H}_{38} \mathrm{NO}_{6}[\mathrm{M}+$ $\mathrm{H}]^{+}$616.2694, found 616.2697.

Diethyl 2'-benzyl-3-(2-(4-bromophenyl)-2-oxoethyl)-2-phenyl2,3-dihydro-2 ${ }^{\prime} H$-spiro[indene-1,3'-isoxazole]-4' ${ }^{\prime}, 5^{\prime}$-dicarboxylate (5eaa), $1.4: 1 \mathrm{dr}$. Purified by silica gel column chromatography (20:1 petroleum ether/ethyl acetate): $125.2 \mathrm{mg}$, 92\% yield; white solid, mp 96-98 ${ }^{\circ} \mathrm{C}$, IR (film) 1735, 1701, 1499, 1373, 1223, $1167 \mathrm{~cm}^{-1} ;{ }^{1} \mathrm{H} \mathrm{NMR}\left(500 \mathrm{MHz}, \mathrm{CDCl}_{3}\right) \delta 7.77(\mathrm{~d}, J=5.0 \mathrm{~Hz}, 1 \mathrm{H})$, $7.65-7.60(\mathrm{~m}, 2 \mathrm{H}), 7.56(\mathrm{~d}, J=10.0 \mathrm{~Hz}, 1 \mathrm{H}), 7.49-7.38(\mathrm{~m}, 4 \mathrm{H})$, $7.33-7.09(\mathrm{~m}, 10 \mathrm{H}), 4.44(\mathrm{t}, J=10.0 \mathrm{~Hz}, 0.4 \mathrm{H}), 4.24-4.10(\mathrm{~m}$, $5.3 \mathrm{H}), 4.03(\mathrm{~d}, J=15.0 \mathrm{~Hz}, 0.6 \mathrm{H}), 3.86(\mathrm{q}, J=10.0 \mathrm{~Hz}, 0.8 \mathrm{H})$, $3.53-3.45(\mathrm{~m}, 1.6 \mathrm{H}), 3.37-3.28(\mathrm{~m}, 1 \mathrm{H}), 3.17(\mathrm{~d}, J=15.0 \mathrm{~Hz}$, $0.4 \mathrm{H}), 1.28-1.20(\mathrm{~m}, 4.3 \mathrm{H}), 1.13(\mathrm{t}, J=10.0 \mathrm{~Hz}, 1.7 \mathrm{H}) ;{ }^{13} \mathrm{C} \mathrm{NMR}$ $\left(125 \mathrm{MHz}, \mathrm{CDCl}_{3}\right) \delta 198.6,198.1,162.7,162.4,159.2,159.2$, $155.6,154.8,147.8,146.7,137.9,137.5,137.4,137.1,136.9$, 136.2 , 135.7, 135.4, 132.1, 132.0, 131.5, 130.5, 130.0, 129.9, 129.7 , 129.7, 128.9, 128.6, 128.6, 128.3, 128.2, 128.0, 127.7, $127.6,127.4,127.3,127.2,127.2$, 126.4, 126.4, 126.1, 124.5, 108.7, 107.2, 85.3, 84.0, 62.6, 62.6, 61.4, 60.9, 60.8, 60.6, 60.2, 58.3, 43.9, 42.4, 42.2, 40.6, 14.2, 14.1, 14.0, 14.0; HRMS(ESI) calcd for $\mathrm{C}_{38} \mathrm{H}_{35} \mathrm{BrNO}_{6}[\mathrm{M}+\mathrm{H}]^{+}$680.1642, found 680.1648 .

Diethyl 2'-benzyl-3-(2-(furan-2-yl)-2-oxoethyl)-2-phenyl-2,3dihydro-2 ${ }^{\prime} H$-spiro[indene-1, $3^{\prime}$-isoxazole $]-4^{\prime}, 5^{\prime}$-dicarboxylate

(5faa), $6.5: 1 \mathrm{dr}$. Purified by silica gel column chromatography (20 : 1 petroleum ether/ethyl acetate): $99.4 \mathrm{mg}, 84 \%$ yield; light yellow solid, mp $65-67{ }^{\circ} \mathrm{C}$, IR (film) 1740, 1710, 1467, 1300, 1253, $1189 \mathrm{~cm}^{-1} ;{ }^{1} \mathrm{H}$ NMR (500 MHz, $\left.\mathrm{CDCl}_{3}\right) \delta 7.53(\mathrm{~s}, 1 \mathrm{H}), 7.43$ $(\mathrm{d}, J=10.0 \mathrm{~Hz}, 2 \mathrm{H}), 7.38-7.07(\mathrm{~m}, 14 \mathrm{H}), 6.48(\mathrm{t}, J=5.0 \mathrm{~Hz}, 1 \mathrm{H})$, $6.42(\mathrm{~s}, 0.1 \mathrm{H}), 4.41(\mathrm{~d}, J=5.0 \mathrm{~Hz}, 1 \mathrm{H}), 4.24-4.17(\mathrm{~m}, 4 \mathrm{H}), 3.88-$ $3.82(\mathrm{~m}, 2 \mathrm{H}), 3.46(\mathrm{~d}, J=15.0 \mathrm{~Hz}, 1 \mathrm{H}), 3.20(\mathrm{dd}, J=20.0,10.0 \mathrm{~Hz}$, $1 \mathrm{H}), 3.09(\mathrm{dd}, J=15.0,5.0 \mathrm{~Hz}, 1 \mathrm{H}), 1.28-1.19(\mathrm{~m}, 5.7 \mathrm{H}), 1.12(\mathrm{t}, J$ $=5.0 \mathrm{~Hz}, 0.4 \mathrm{H}) ;{ }^{13} \mathrm{C} \mathrm{NMR}\left(125 \mathrm{MHz}, \mathrm{CDCl}_{3}\right) \delta$ 188.5, 162.7, 
$159.2,155.5,152.8,146.7,146.6,137.4,136.9,135.4,130.4$, $130.0,129.0,128.6,128.2$, 127.9, 127.5, 127.2, 127.1, 126.0, 124.6, 117.4, 112.4, 107.2, 84.0, 62.6, 61.5, 60.8, 60.2, 41.9, 40.4, 29.9, 14.2, 14.0; HRMS(ESI) calcd for $\mathrm{C}_{36} \mathrm{H}_{34} \mathrm{NO}_{7}[\mathrm{M}+\mathrm{H}]^{+}$ 592.2330, found 592.2333.

Diethyl 2'-benzyl-3-(2-oxopropyl)-2-phenyl-2,3-dihydro-2 ${ }^{\prime} H$ spiro-[indene-1, $3^{\prime}$-is-oxazole]- $4^{\prime}, 5^{\prime}$-dicarboxylate (5gaa), $13.0: 1$ dr. Purified by silica gel column chromatography $(30: 1$ petroleum ether/ethyl acetate): $82.0 \mathrm{mg}, 76 \%$ yield; Light yellow oil, IR (film) 1712, 1685, 1448, 1370, 1223, $1108 \mathrm{~cm}^{-1}$; ${ }^{1} \mathrm{H}$ NMR (400 $\left.\mathrm{MHz}, \mathrm{CDCl}_{3}\right) \delta 7.62(\mathrm{~d}, J=4.0 \mathrm{~Hz}, 2 \mathrm{H}), 7.45-7.21(\mathrm{~m}, 12 \mathrm{H}), 4.20-$ 4.05 (m, 5H), 4.01-3.84 (m, 2H), 3.52-3.42 (m, 1H), 3.03-2.98 (m, 1H), 2.88-2.81 (m, 1H), 2.75 (d, $J=4.0 \mathrm{~Hz}, 0.1 \mathrm{H}), 2.15$ (s, $0.2 \mathrm{H}), 2.02(\mathrm{~s}, 2.8 \mathrm{H}), 1.29-1.19(\mathrm{~m}, 3.2 \mathrm{H}), 1.12(\mathrm{~d}, J=8.0 \mathrm{~Hz}$, $2.8 \mathrm{H}) ;{ }^{13} \mathrm{C} \mathrm{NMR}\left(100 \mathrm{MHz}, \mathrm{CDCl}_{3}\right) \delta 207.7,162.4,159.2,154.7$, $147.9,137.6,137.2,137.1,131.7,129.7,129.0,128.6,128.1$, 127.6, 127.4, 127.2, 126.4, 126.2, 108.7, 85.2, 62.6, 60.9, 60.5, 58.0, 47.4, 43.4, 31.0, 14.1, 13.9. HRMS (ESI) calcd for $\mathrm{C}_{33} \mathrm{H}_{33} \mathrm{NO}_{6} \mathrm{Na}[\mathrm{M}+\mathrm{Na}]^{+}$562.2200, found 562.2199.

Diethyl 2'-benzyl-3-(2-oxo-2-( $p$-tolyl)ethyl)-2-( $m$-tolyl)-2,3dihydro- $2^{\prime} H$-spiro[indene- $1,3^{\prime}$-isoxazole]- $4^{\prime}, 5^{\prime}$-dicarboxylate

(5haa), $1.9: 1$ dr. Purified by silica gel column chromatography (20:1 petroleum ether/ethyl acetate): $112.1 \mathrm{mg}, 89 \%$ yield; white yellow solid, mp 50-52 ${ }^{\circ} \mathrm{C}$, IR (film) 1741, 1712, 1496, 1370, 1300, 1241, $1140 \mathrm{~cm}^{-1} ;{ }^{1} \mathrm{H}$ NMR (400 MHz, $\left.\mathrm{CDCl}_{3}\right) \delta 7.84$ $(\mathrm{d}, J=8.0 \mathrm{~Hz}, 0.7 \mathrm{H}), 7.67(\mathrm{~d}, J=8.0 \mathrm{~Hz}, 1.3 \mathrm{H}), 7.55(\mathrm{~d}, J=$ $8.0 \mathrm{~Hz}, 0.7 \mathrm{H}), 7.45-7.27(\mathrm{~m}, 7.6 \mathrm{H}), 7.24-7.11(\mathrm{~m}, 5.9 \mathrm{H}), 7.06$ (d, $=8.0 \mathrm{~Hz}, 0.4 \mathrm{H}), 7.01(\mathrm{~d}, J=8.0 \mathrm{~Hz}, 0.7 \mathrm{H}), 4.48(\mathrm{t}, J=8.0 \mathrm{~Hz}$, $0.3 \mathrm{H}), 4.26-4.07(\mathrm{~m}, 5.1 \mathrm{H}), 4.02(\mathrm{~d}, J=16.0 \mathrm{~Hz}, 0.8 \mathrm{H}), 3.85(\mathrm{dd}, J$ $=16.0,8.0 \mathrm{~Hz}, 0.7 \mathrm{H}), 3.55-3.45(\mathrm{~m}, 1.6 \mathrm{H}), 3.34(\mathrm{dd}, J=20.0$, $8.0 \mathrm{~Hz}, 1 \mathrm{H}), 3.20$ (dd, $J=20.0,4.0 \mathrm{~Hz}, 0.4 \mathrm{H}), 2.39(\mathrm{~s}, 0.9 \mathrm{H}), 2.36$ $(\mathrm{s}, 1.9 \mathrm{H}), 2.32(\mathrm{~s}, 1.1 \mathrm{H}), 2.21(\mathrm{~s}, 2 \mathrm{H}), 1.30-1.19(\mathrm{~m}, 4.1 \mathrm{H}), 1.12(\mathrm{t}$, $J=8.0 \mathrm{~Hz}, 1.9 \mathrm{H}) ;{ }^{13} \mathrm{C} \mathrm{NMR}\left(100 \mathrm{MHz}, \mathrm{CDCl}_{3}\right) \delta 199.3,198.8$, $162.8,162.4,159.4,159.3,155.6,154.8,148.0,147.0,144.2$, $143.8,138.0,137.6,137.5,137.4,137.2$, 137.1, 135.5, 135.0, $134.6,132.6,131.3,129.9,129.6,129.4,129.3,128.8,128.5$, $128.5,128.3,128.2,128.1,127.9,127.9,127.5$, 127.2, 127.1, 127.0, 126.4, 126.2, 125.9, 124.8, 108.8, 107.2, 85.2, 83.9, 62.6, $61.1,60.8,60.7,60.6,60.2,58.2,44.0,42.4,42.3,40.4,29.9,21.8$, $21.8,21.7,21.6,14.2,14.1,14.1,14.0$; HRMS (ESI) calcd for $\mathrm{C}_{40} \mathrm{H}_{39} \mathrm{NO}_{6} \mathrm{Na}[\mathrm{M}+\mathrm{Na}]^{+}$652.2670, found 652.2669.

Diethyl 2'-benzyl-3-(2-oxo-2-( $p$-tolyl)ethyl)-2-( $p$-tolyl)-2,3-dihydro- $2^{\prime} H$-spiro[indene-1, $3^{\prime}$-isoxazole $]-4^{\prime}, 5^{\prime}$-dicarboxylate (5iaa), $2.2: 1 \mathrm{dr}$. Purified by silica gel column chromatography (20:1 petroleum ether/ethyl acetate): $117.1 \mathrm{mg}$, 93\% yield; light yellow solid, mp 55-57 ${ }^{\circ} \mathrm{C}$, IR (film) 1739, 1709, 1497, 1371, 1302, 1241, $1140 \mathrm{~cm}^{-1} ;{ }^{1} \mathrm{H}$ NMR (400 MHz, $\left.\mathrm{CDCl}_{3}\right) \delta 7.83(\mathrm{~d}, J=8.0 \mathrm{~Hz}$, $1.5 \mathrm{H}), 7.69(\mathrm{~d}, J=8.0 \mathrm{~Hz}, 0.5 \mathrm{H}), 7.55(\mathrm{~d}, J=8.0 \mathrm{~Hz}, 0.6 \mathrm{H}), 7.44-$ $7.39(\mathrm{~m}, 0.7 \mathrm{H}), 7.37-7.32(\mathrm{~m}, 3.3 \mathrm{H}), 7.29-7.09(\mathrm{~m}, 10 \mathrm{H}), 7.05(\mathrm{~d}$, $J=8.0 \mathrm{~Hz}, 0.6 \mathrm{H}), 4.47(\mathrm{t}, J=8.0 \mathrm{~Hz}, 0.8 \mathrm{H}), 4.25-4.07(\mathrm{~m}, 4.5 \mathrm{H})$, $4.01(\mathrm{~d}, J=12.0 \mathrm{~Hz}, 0.3 \mathrm{H}), 3.84(\mathrm{dd}, J=20.0,12.0 \mathrm{~Hz}, 1.5 \mathrm{H})$, $3.53-3.38$ (m, 1.5H), 3.31 (dd, $J=16.0,8.0 \mathrm{~Hz}, 0.8 \mathrm{H}), 3.20(\mathrm{dd}, J$ $=16.0,4.0 \mathrm{~Hz}, 0.8 \mathrm{H}), 2.39(\mathrm{~s}, 2.1 \mathrm{H}), 2.36(\mathrm{~s}, 0.9 \mathrm{H}), 2.30(\mathrm{~s}, 2.1 \mathrm{H})$, $2.27(\mathrm{~s}, 0.9 \mathrm{H}), 1.29-1.19(\mathrm{~m}, 5.1 \mathrm{H}), 1.12(\mathrm{t}, J=8.0 \mathrm{~Hz}, 0.9 \mathrm{H}) ;{ }^{13} \mathrm{C}$ NMR $\left(100 \mathrm{MHz}, \mathrm{CDCl}_{3}\right) \delta 199.3,198.9,162.8,162.4,159.3,155.3$, $154.7,148.1,147.2,144.2$, 143.8, 137.8, 137.5, 137.1, 137.0, $136.7,135.1,134.6,134.1,132.5$, 131.5, 130.4, 129.9, 129.6,
$129.4,129.3,129.0,128.9,128.8,128.6,128.6,128.5,128.3$, 128.3 , 127.6, 127.2, 127.1, 127.0, 126.6, 126.3, 126.0, 124.7, 108.9, 107.5, 85.3, 84.0, 62.5, 61.1, 60.8, 60.6, 60.3, 58.1, 44.0, 42.5, 42.1, 40.5, 29.9, 21.8, 21.4, 21.3, 21.2, 14.2, 14.1, 14.0, 14.0; HRMS(ESI) calcd for $\mathrm{C}_{40} \mathrm{H}_{40} \mathrm{NO}_{6}[\mathrm{M}+\mathrm{H}]^{+} 630.2850$, found 630.2852 .

Diethyl 2'-benzyl-2-(4-chlorophenyl)-3-(2-oxo-2-( $p$-tolyl)ethyl)2,3-dihydro-2 ${ }^{\prime} H$-spiro[indene-1,3'-isoxazole]-4', $5^{\prime}$-dicarboxylate

(5jaa), $1.6: 1$ dr. Purified by silica gel column chromatography (20: 1 petroleum ether/ethyl acetate): $127.4 \mathrm{mg}$, 98\% yield; light yellow solid, mp 95-97 ${ }^{\circ} \mathrm{C}$, IR (film) 1741, 1701, 1476, 1353, 1226, $1156 \mathrm{~cm}^{-1} ;{ }^{1} \mathrm{H}$ NMR (400 MHz, $\left.\mathrm{CDCl}_{3}\right) \delta 7.83$ (d, $J=$ $8.0 \mathrm{~Hz}, 0.7 \mathrm{H}), 7.70(\mathrm{~d}, J=8.0 \mathrm{~Hz}, 1.2 \mathrm{H}), 7.60(\mathrm{~d}, J=8.0 \mathrm{~Hz}$, $1.3 \mathrm{H}), 7.44-7.37(\mathrm{~m}, 2.6 \mathrm{H}), 7.34-7.28(\mathrm{~m}, 5 \mathrm{H}), 7.25-7.11(\mathrm{~m}$, $6.4 \mathrm{H}), 4.44(\mathrm{t}, J=8.0 \mathrm{~Hz}, 0.4 \mathrm{H}), 4.25-4.08(\mathrm{~m}, 5.2 \mathrm{H}), 4.01(\mathrm{~d}, J=$ $12.0 \mathrm{~Hz}, 0.7 \mathrm{H}), 3.88(\mathrm{~d}, J=16.0 \mathrm{~Hz}, 0.4 \mathrm{H}), 3.79(\mathrm{~d}, J=12.0 \mathrm{~Hz}$, $0.4 \mathrm{H}), 3.52-3.45(\mathrm{~m}, 1.5 \mathrm{H}), 3.42-3.31(\mathrm{~m}, 1.2 \mathrm{H}), 3.13(\mathrm{dd}, J=$ 16.0, $4.0 \mathrm{~Hz}, 0.4 \mathrm{H}), 2.40(\mathrm{~s}, 1.2 \mathrm{H}), 2.38(\mathrm{~s}, 1.8 \mathrm{H}), 1.30-1.20(\mathrm{~m}$, $4.2 \mathrm{H}), 1.13(\mathrm{t}, J=8.0 \mathrm{~Hz}, 1.8 \mathrm{H}) ;{ }^{13} \mathrm{C} \mathrm{NMR}\left(100 \mathrm{MHz}, \mathrm{CDCl}_{3}\right.$ ) $\delta 199.0,198.5,162.7,162.3,159.1,155.4,154.8,147.9,146.8$, $144.3,144.0,137.3,137.2,137.1,136.8,135.7,134.9,134.4$, $134.1,133.3,133.3,132.9,131.8,130.1,129.8,129.5,129.4$, $129.0,128.6$, 128.4, 128.3, 128.3, 128.2, 128.1, 127.7, 127.4, 127.3, 127.2 , 126.6, 126.3, 126.0, 124.7, 108.5, 107.2, 100.1, 85.2, 84.0, 62.8, 61.0, 60.6, 60.2, 57.6, 43.8, 42.4, 41.9, 40.7, 21.8, 14.2, 14.1, 14.0, 14.0; HRMS (ESI) calcd for $\mathrm{C}_{39} \mathrm{H}_{36} \mathrm{NO}_{6} \mathrm{ClNa}[\mathrm{M}+\mathrm{Na}]^{+}$ 672.2123, found 672.2128.

Diethyl 2'-benzyl-2-(4-bromophenyl)-3-(2-oxo-2-( $p$-tolyl) ethyl)-2,3-dihydro- $2^{\prime} H$-spiro[indene-1,3'-isoxazole]-4' ${ }^{\prime}, 5^{\prime}$-dicarboxylate (5kaa), 1.1 : $1 \mathrm{dr}$. Purified by silica gel column chromatography (20:1 petroleum ether/ethyl acetate): $126.4 \mathrm{mg}$, 91\% yield; white solid, mp 82-84 ${ }^{\circ} \mathrm{C}$, IR (film) 1749, 1712, 1488, 1371, 1299, $1180 \mathrm{~cm}^{-1}$; ${ }^{1} \mathrm{H}$ NMR (400 MHz, $\left.\mathrm{CDCl}_{3}\right) \delta 7.82(\mathrm{~d}, J=$ $8.0 \mathrm{~Hz}, 1 \mathrm{H}), 7.69$ (d, $J=8.0 \mathrm{~Hz}, 1 \mathrm{H}), 7.54(\mathrm{~d}, J=8.0 \mathrm{~Hz}, 1 \mathrm{H})$, $7.44-7.27(\mathrm{~m}, 8 \mathrm{H}), 7.25-7.10(\mathrm{~m}, 6 \mathrm{H}), 4.43(\mathrm{t}, J=8.0 \mathrm{~Hz}, 0.5 \mathrm{H})$, 4.25-4.15 (m, 3H), 4.13-4.07 (m, $1.8 \mathrm{H}), 4.00(\mathrm{~d}, J=16.0 \mathrm{~Hz}$, $0.5 \mathrm{H}), 3.88(\mathrm{~d}, J=12.0 \mathrm{~Hz}, 0.6 \mathrm{H}), 3.78(\mathrm{~d}, J=12.0 \mathrm{~Hz}, 0.6 \mathrm{H})$, $3.52-3.30(\mathrm{~m}, 2.5 \mathrm{H}), 3.12(\mathrm{dd}, J=16.0,4.0 \mathrm{~Hz}, 0.6 \mathrm{H}), 2.40(\mathrm{~s}$, $1.5 \mathrm{H}), 2.38(\mathrm{~s}, 1.5 \mathrm{H}), 1.30-1.19(\mathrm{~m}, 4.4 \mathrm{H}), 1.13(\mathrm{t}, J=8.0 \mathrm{~Hz}$, $1.6 \mathrm{H}) ;{ }^{13} \mathrm{C} \mathrm{NMR}\left(100 \mathrm{MHz}, \mathrm{CDCl}_{3}\right) \delta 199.0,198.5,162.7,162.3$, $159.1,155.4,154.8,147.9,146.8,144.3,144.0,137.2,137.1$, $136.8,136.2$, 135.0, 134.7, 134.5, 133.3, 132.2, 131.3, 131.1, $130.1,129.8,129.5,129.4,129.0,128.7,128.6,128.5,128.3$, $128.3,127.7,127.4,127.3,127.2$, 126.6, 126.3, 126.0, 124.7, 121.6, 121.6, 108.5, 107.2, 85.3, 84.0, 62.8, 62.7, 61.1, 61.0, 60.6, 60.3, 57.7, 43.8, 42.4, 41.9, 40.7, 29.9, 21.9, 21.8, 14.2, 14.1, 14.0, 14.0; HRMS(ESI) calcd for $\mathrm{C}_{39} \mathrm{H}_{37} \mathrm{O}_{6} \mathrm{BrN}[\mathrm{M}+\mathrm{H}]{ }^{+} 694.1799$; found: 694.1799 .

Diethyl 2'-benzyl-2-(4-methoxyphenyl)-3-(2-oxo-2-( $p$-tolyl) ethyl)-2,3-dihydro-2' $H$-spiro[indene-1,3'-isoxazole $]-4^{\prime}, 5^{\prime}$-dicarboxylate (5laa), 6.5 : $1 \mathrm{dr}$. Purified by silica gel column chromatography (20:1 petroleum ether/ethyl acetate): $127.9 \mathrm{mg}$, 99\% yield; light yellow solid, mp 78-80 ${ }^{\circ} \mathrm{C}$, IR (film) 1739, 1711, 1491, 1370, 1300, $1107 \mathrm{~cm}^{-1} ;{ }^{1} \mathrm{H}$ NMR (400 MHz, $\left.\mathrm{CDCl}_{3}\right) \delta 7.84$ $(\mathrm{d}, J=8.0 \mathrm{~Hz}, 0.2 \mathrm{H}), 7.71(\mathrm{~d}, J=8.0 \mathrm{~Hz}, 1.8 \mathrm{H}), 7.59(\mathrm{~d}, J=$ $8.0 \mathrm{~Hz}, 2 \mathrm{H}), 7.44-7.37(\mathrm{~m}, 2 \mathrm{H}), 7.34-7.21(\mathrm{~m}, 7 \mathrm{H}), 7.17(\mathrm{t}, J=$ $8.0 \mathrm{~Hz}, 2 \mathrm{H}), 6.83(\mathrm{~d}, J=8.0 \mathrm{~Hz}, 0.2 \mathrm{H}), 6.78(\mathrm{~d}, J=8.0 \mathrm{~Hz}, 1.8 \mathrm{H})$, 
4.22-4.05 (m, 5.9H), $4.01(\mathrm{~d}, J=12.0 \mathrm{~Hz}, 0.9 \mathrm{H}), 3.77(\mathrm{~s}, 0.4 \mathrm{H})$, $3.75(\mathrm{~s}, 2.6 \mathrm{H}), 3.52-3.38(\mathrm{~m}, 2.8 \mathrm{H}), 2.40(\mathrm{~s}, 0.4 \mathrm{H}), 2.37(\mathrm{~s}, 2.6 \mathrm{H})$, 1.30-1.19 (m, 3.4H), $1.13(\mathrm{t}, J=8.0 \mathrm{~Hz}, 2.6 \mathrm{H}) ;{ }^{13} \mathrm{C}$ NMR $(100$ $\left.\mathrm{MHz}_{\mathrm{CDCl}}\right) \delta 198.9,162.4,159.2,158.8,154.6,148.1,143.9$, $137.7,137.4,135.0,132.7,131.5,129.6,129.3$, 129.2, 129.0, $128.6,128.6$, 128.5, 128.3, 127.6, 127.1, 126.6, 126.3, 113.5, 108.9, 85.3, 62.6, 60.9, 60.6, 57.7, 55.3, 44.0, 42.5, 40.8, 21.8, 14.1, 14.0; HRMS (ESI) calcd for $\mathrm{C}_{40} \mathrm{H}_{39} \mathrm{NO}_{7} \mathrm{Na}[\mathrm{M}+\mathrm{Na}]^{+}$ 668.2619, found 668.2615.

Diethyl 2'-benzyl-2-(cyclohex-1-en-1-yl)-3-(2-oxo-2-( $p$-tolyl) ethyl)-2,3-dihydro- ${ }^{\prime} H$-spiro[indene-1,3'-isoxazole]- $4^{\prime}, 5^{\prime}$-dicarboxylate (5maa), $6.8: 1 \mathrm{dr}$. Purified by silica gel column chromatography (40:1 petroleum ether/ethyl acetate): $114.0 \mathrm{mg}$, $92 \%$ yield; light yellow solid, mp $85-87^{\circ} \mathrm{C}$, IR (film) 1733, 1710, 1372, 1298, 1200, $1175 \mathrm{~cm}^{-1} ;{ }^{1} \mathrm{H}$ NMR (500 MHz, $\left.\mathrm{CDCl}_{3}\right) \delta 7.95$ $(\mathrm{d}, J=10.0 \mathrm{~Hz}, 0.2 \mathrm{H}), 7.81(\mathrm{~d}, J=10.0 \mathrm{~Hz}, 1.7 \mathrm{H}), 7.39(\mathrm{~d}, J=$ $5.0 \mathrm{~Hz}, 1 \mathrm{H}), 7.34-7.23(\mathrm{~m}, 8.2 \mathrm{H}), 7.18(\mathrm{~d}, J=10.0 \mathrm{~Hz}, 1.8 \mathrm{H}), 6.22$ $(\mathrm{s}, 0.8 \mathrm{H}), 4.34-4.28(\mathrm{~m}, 2 \mathrm{H}), 4.16-4.06(\mathrm{~m}, 2.8 \mathrm{H}), 3.99(\mathrm{~d}, J=$ $15.0 \mathrm{~Hz}, 0.8 \mathrm{H}), 3.81-3.76(\mathrm{~m}, 0.9 \mathrm{H}), 3.41$ (dd, $J=15.0,10.0 \mathrm{~Hz}$, $0.9 \mathrm{H}), 3.32-3.20(\mathrm{~m}, 1.1 \mathrm{H}), 3.01$ (dd, $J=15.0,5.0 \mathrm{~Hz}, 0.8 \mathrm{H}), 2.43$ $(\mathrm{s}, 0.4 \mathrm{H}), 2.39(\mathrm{~s}, 2.6 \mathrm{H}), 2.26-2.13(\mathrm{~m}, 0.9 \mathrm{H}), 2.04-1.99(\mathrm{~m}$, $0.3 \mathrm{H}), 1.90-1.82(\mathrm{~m}, 1.6 \mathrm{H}), 1.62-1.56(\mathrm{~m}, 2.8 \mathrm{H}), 1.49-1.40$ (m, $2.7 \mathrm{H}), 1.36-1.33(\mathrm{~m}, 3 \mathrm{H}), 1.17(\mathrm{~d}, J=10.0 \mathrm{~Hz}, 0.4 \mathrm{H}), 1.13(\mathrm{~d}, J=$ $10.0 \mathrm{~Hz}, 2.6 \mathrm{H}) ;{ }^{13} \mathrm{C} \mathrm{NMR}\left(100 \mathrm{MHz}, \mathrm{CDCl}_{3}\right) \delta$ 199.4, 198.6, 162.7, $162.5,160.0,159.8,155.3,148.1,147.1,144.2,143.7,137.7$, $137.6,137.4,135.1,134.8,133.2,132.6,129.8,129.5,129.3$, $128.8,128.6,128.6,128.5,128.4,128.3,127.5,127.3,126.9$, 126.8, 126.1, 126.0, 125.8, 124.6, 108.1, 85.0, 84.4, 62.7, 60.8, 60.6, 59.9, 58.2 , 42.6, 42.3, 42.0, 38.4, 31.5, 29.9, 28.5, 27.8, 27.1, 25.9, 25.8, 23.5, 23.4, 22.6, 22.2, 21.9, 21.8, 14.2, 14.1; HRMS (ESI) calcd for $\mathrm{C}_{39} \mathrm{H}_{41} \mathrm{NO}_{6} \mathrm{Na}[\mathrm{M}+\mathrm{Na}]^{+}$642.2826, found 642.2823.

Diethyl 2'-benzyl-5-fluoro-3-(2-oxo-2-( $p$-tolyl)ethyl)-2-phenyl2,3-dihydro-2 ${ }^{\prime} H$-spiro[indene-1,3'-isoxazole]- $4^{\prime}, 5^{\prime}$-dicarboxylate (5naa), $4.5: 1$ dr. Purified by silica gel column chromatography (20:1 petroleum ether/ethyl acetate): $119.1 \mathrm{mg}$, 94\% yield; yellowish white solid, mp $116-118^{\circ} \mathrm{C}$, IR (film) 1743, 1709, 1474, 1303, 1231, $1181 \mathrm{~cm}^{-1}$; ${ }^{1} \mathrm{H}$ NMR (400 MHz, $\left.\mathrm{CDCl}_{3}\right) \delta 7.83(\mathrm{~d}, J=$ $8.0 \mathrm{~Hz}, 0.4 \mathrm{H}), 7.71$ (d, $J=8.0 \mathrm{~Hz}, 1.6 \mathrm{H}), 7.65$ (d, $J=8.0 \mathrm{~Hz}$, $1.6 \mathrm{H}), 7.42-7.36(\mathrm{~m}, 1.4 \mathrm{H}), 7.35-7.14(\mathrm{~m}, 11 \mathrm{H}), 7.02-6.97(\mathrm{~m}$, $1 \mathrm{H}), 6.82(\mathrm{~d}, J=8.0 \mathrm{~Hz}, 0.2 \mathrm{H}), 4.45(\mathrm{t}, J=8.0 \mathrm{~Hz}, 0.2 \mathrm{H}), 4.30-$ 4.07 (m, 5.6H), 3.99 (d, $J=12.0 \mathrm{~Hz}, 0.8 \mathrm{H}), 3.87$ (d, $J=12.0 \mathrm{~Hz}$, $0.4 \mathrm{H}), 3.53-3.40(\mathrm{~m}, 2.6 \mathrm{H}), 3.33-3.14(\mathrm{~m}, 0.4 \mathrm{H}), 2.40(\mathrm{~s}, 0.5 \mathrm{H})$, $2.38(\mathrm{~s}, 2.5 \mathrm{H}), 1.29-1.22(\mathrm{~m}, 3.5 \mathrm{H}), 1.15(\mathrm{t}, J=8.0 \mathrm{~Hz}, 2.5 \mathrm{H}) ;{ }^{13} \mathrm{C}$ NMR (100 MHz, $\mathrm{CDCl}_{3}$ ) $\delta 198.8,198.4,168.4,163.7$ (d, $J=246.8$ Hz), 162.7, 162.3, 159.2, 159.1, 155.7, 155.0, 150.6, 150.5, 149.4, 144.4, 144.1, 141.1, 137.2, 136.8, 136.7, 135.2, 134.8, 134.3, 133.5 $(\mathrm{d}, J=2.6 \mathrm{~Hz}), 131.6,130.4,130.2,129.5,129.4,129.0,128.6$, 128.6 , 128.5, 128.3, 128.3, 128.1, 127.7, 127.6, 127.5, 127.5, 127.3, 120.5, 114.6 (d, $J=22.9 \mathrm{~Hz}), 114.0(\mathrm{~d}, J=22.4 \mathrm{~Hz}), 112.4$, 112.2, 108.2, 106.8, 92.9, 84.6, 84.0, 83.3, 62.7, 61.6, 61.0, 60.5, 60.1, 58.8, 43.7, 42.1, 41.9, 40.4, 29.9, 21.8, 14.2, 14.1, 14.0, 14.0; ${ }^{19} \mathrm{~F}$ NMR $\left(282 \mathrm{MHz}, \mathrm{CDCl}_{3}\right): \delta-111.5(\mathrm{~s}, 1 \mathrm{~F})$; HRMS(ESI) calcd for $\mathrm{C}_{39} \mathrm{H}_{37} \mathrm{FNO}_{6}[\mathrm{M}+\mathrm{H}]^{+}$634.2599, found 634.2603.

Diethyl 2'-benzyl-5-chloro-3-(2-oxo-2-( $p$-tolyl)ethyl)-2-phenyl2,3-dihydro-2 ${ }^{\prime} H$-spiro[indene-1,3'-isoxazole]- $4^{\prime}, 5^{\prime}$-dicarboxylate (5oaa), 2.5 : 1 dr. Purified by silica gel column chromatography
(20: 1 petroleum ether/ethyl acetate): $117.0 \mathrm{mg}$, 90\% yield; light yellow solid, mp 112-114 ${ }^{\circ} \mathrm{C}$, IR (film) 1740, 1709, 1466, 1304, 1261, $1180 \mathrm{~cm}^{-1} ;{ }^{1} \mathrm{H}$ NMR $\left(400 \mathrm{MHz}, \mathrm{CDCl}_{3}\right) \delta 7.83(\mathrm{~d}, J=$ $8.0 \mathrm{~Hz}, 1.4 \mathrm{H}), 7.71(\mathrm{~d}, J=12.0 \mathrm{~Hz}, 0.5 \mathrm{H}), 7.64(\mathrm{~d}, J=4.0 \mathrm{~Hz}$, $0.5 \mathrm{H}), 7.47(\mathrm{~d}, J=1.6 \mathrm{~Hz}, 0.3 \mathrm{H}), 7.42(\mathrm{~d}, J=8.0 \mathrm{~Hz}, 1.5 \mathrm{H}), 7.39-$ $7.10(\mathrm{~m}, 13 \mathrm{H}), 4.45(\mathrm{t}, J=8.0 \mathrm{~Hz}, 0.7 \mathrm{H}), 4.26-4.09(\mathrm{~m}, 4.4 \mathrm{H}), 3.99$ $(\mathrm{d}, J=12.0 \mathrm{~Hz}, 0.3 \mathrm{H}), 3.89-3.84(\mathrm{~m}, 1.5 \mathrm{H}), 3.52-3.42(\mathrm{~m}, 1.5 \mathrm{H})$, $3.33-3.18(\mathrm{~m}, 1.6 \mathrm{H}), 2.40(\mathrm{~s}, 2 \mathrm{H}), 2.38(\mathrm{~s}, 1 \mathrm{H}), 1.29-1.21(\mathrm{~m}$, $5.1 \mathrm{H}), 1.16(\mathrm{t}, J=8.0 \mathrm{~Hz}, 0.9 \mathrm{H}) ;{ }^{13} \mathrm{C} \mathrm{NMR}\left(100 \mathrm{MHz}, \mathrm{CDCl}_{3}\right)$ $\delta 198.7,198.3,162.6,162.3,159.1,155.8,149.9,149.0,144.4$, $144.1,137.1,136.7,136.6,136.5,136.1,135.9,135.7,135.1$, $134.8,134.3,131.5,130.4,129.5,129.4,129.0,128.6,128.6$, $128.5,128.3,128.3,128.3,128.1,127.7,127.6,127.5,127.4$, 127.3 127.0, 125.3, 108.1, 106.6, 84.7, 83.4, 62.7, 61.3, 60.9, 60.6, 60.2 , 58.5, 43.6, 42.0, 41.9, 40.3, 29.9, 21.8, 14.2, 14.1, 14.0, 14.0; HRMS(ESI) calcd for $\mathrm{C}_{39} \mathrm{H}_{37} \mathrm{ClNO}_{6}[\mathrm{M}+\mathrm{H}]^{+}$650.2304, found 650.2308 .

Diethyl 2'-benzyl-7-(2-oxo-2-( $p$-tolyl)ethyl)-6-phenyl-6,7-dihydro-2 ${ }^{\prime} H$-spiro[indeno-[5,6-d][1,3]dioxole-5, $3^{\prime}$-isoxazole $]-4^{\prime}, 5^{\prime}$ -

dicarboxylate (5paa), $3.2: 1 \mathrm{dr}$. Purified by silica gel column chromatography (10:1 petroleum ether/ethyl acetate): $106.9 \mathrm{mg}, 81 \%$ yield; yellowish white solid, mp $149-151{ }^{\circ} \mathrm{C}$, IR (film) 1734, 1705, 1497, 1370, 1258, $1143 \mathrm{~cm}^{-1} ;{ }^{1} \mathrm{H}$ NMR (400 $\left.\mathrm{MHz} \mathrm{CDCl}_{3}\right) \delta 7.82(\mathrm{~d}, J=12.0 \mathrm{~Hz}, 1.6 \mathrm{H}), 7.71-7.65(\mathrm{~m}, 1 \mathrm{H})$, $7.42(\mathrm{~d}, J=8.0 \mathrm{~Hz}, 1.6 \mathrm{H}), 7.35(\mathrm{t}, J=4.0 \mathrm{~Hz}, 1.3 \mathrm{H}), 7.31-7.15(\mathrm{~m}$, $9 \mathrm{H}), 6.86(\mathrm{~d}, J=8.0 \mathrm{~Hz}, 0.5 \mathrm{H}), 6.80(\mathrm{~s}, 0.7 \mathrm{H}), 6.60(\mathrm{~s}, 0.7 \mathrm{H}), 5.97-$ $5.93(\mathrm{~m}, 1.9 \mathrm{H}), 4.40-4.35(\mathrm{~m}, 0.9 \mathrm{H}), 4.27-4.12(\mathrm{~m}, 4.2 \mathrm{H}), 4.03-$ $3.95(\mathrm{~m}, 0.5 \mathrm{H}), 3.91-3.83(\mathrm{~m}, 1.4 \mathrm{H}), 3.57(\mathrm{dd}, J=20.0,12.0 \mathrm{~Hz}$, $1 \mathrm{H}), 3.41$ (d, $J=8.0 \mathrm{~Hz}, 0.4 \mathrm{H}), 3.27$ (dd, $J=16.0,8.0 \mathrm{~Hz}, 0.8 \mathrm{H}$ ), $3.16(\mathrm{dd}, J=16.0,4.0 \mathrm{~Hz}, 0.7 \mathrm{H}), 2.39(\mathrm{~s}, 2.3 \mathrm{H}), 2.37(\mathrm{~s}, 0.7 \mathrm{H})$, 1.29-1.21 (m, 5.3H), $1.18(\mathrm{t}, J=8.0 \mathrm{~Hz}, 0.7 \mathrm{H}) ;{ }^{13} \mathrm{C}$ NMR $(100$ $\left.\mathrm{MHz}, \mathrm{CDCl}_{3}\right) \delta 199.2,198.9,162.8,162.4,159.3,155.5,154.8$, $149.4,149.1,147.3,147.2,144.3,143.9,142.0,140.9,137.5$, $137.2,137.0,136.1,135.7,135.0,134.5,131.6,130.5,130.3$, $129.5,129.4,129.0,128.9,128.6,128.5,128.5,128.3,128.3$, 128.2 , 128.0, 127.6, 127.5, 127.3, 127.2, 107.0, 106.9, 106.0, 105.9, 105.5, 101.6, 85.2, 83.9, 62.6, 61.5, 60.9, 60.9, 60.6, 60.0, $58.9,43.7,42.6,42.4,40.2,21.8,14.3,14.2,14.0,14.0$; HRMS(ESI) calcd for $\mathrm{C}_{40} \mathrm{H}_{37} \mathrm{NO}_{8} \mathrm{Na}[\mathrm{M}+\mathrm{Na}]^{+} 682.2411$, found 682.2406 .

Diethyl 2'-benzyl-3-(2-oxo-2-( $p$-tolyl)ethyl)-2-phenyl-2,3-dihydro- $2^{\prime} H$-spiro[cyclopenta[ $\left.a\right]$ naphthalene-1, $3^{\prime}$-isoxazole $]-4^{\prime}, 5^{\prime}$ -

dicarboxylate (5qaa), $21.0: 1 \mathrm{dr}$. Purified by silica gel column chromatography (40:1 petroleum ether/ethyl acetate): $115.8 \mathrm{mg}, 87 \%$ yield; yellowish white solid, $\mathrm{mp} 158-160{ }^{\circ} \mathrm{C}$, IR (film) 1747, 1711, 1370, 1300, 1181, $1094 \mathrm{~cm}^{-1} ;{ }^{1} \mathrm{H}$ NMR (400 $\left.\mathrm{MHz}, \mathrm{CDCl}_{3}\right) \delta 8.34(\mathrm{~d}, J=8.0 \mathrm{~Hz}, 1 \mathrm{H}), 7.86(\mathrm{~d}, J=8.0 \mathrm{~Hz}, 1 \mathrm{H})$, $7.79(\mathrm{~d}, J=8.0 \mathrm{~Hz}, 1 \mathrm{H}), 7.70(\mathrm{t}, J=8.0 \mathrm{~Hz}, 4 \mathrm{H}), 7.55(\mathrm{t}, J=8.0 \mathrm{~Hz}$, $2 \mathrm{H}), 7.45(\mathrm{t}, J=8.0 \mathrm{~Hz}, 1 \mathrm{H}), 7.32-7.20(\mathrm{~m}, 8 \mathrm{H}), 7.15(\mathrm{~d}, J=$ $8.0 \mathrm{~Hz}, 2 \mathrm{H}), 4.30-4.24(\mathrm{~m}, 3 \mathrm{H}), 4.20(\mathrm{~d}, J=8.0 \mathrm{~Hz}, 2 \mathrm{H}), 4.05-3.90$ $(\mathrm{m}, 2 \mathrm{H}), 3.75-3.55(\mathrm{~m}, 3 \mathrm{H}), 2.37(\mathrm{~s}, 3 \mathrm{H}), 1.23(\mathrm{t}, J=8.0 \mathrm{~Hz}, 3 \mathrm{H})$, $0.98(\mathrm{~d}, J=8.0 \mathrm{~Hz}, 0.1 \mathrm{H}), 0.85(\mathrm{~d}, J=8.0 \mathrm{~Hz}, 3 \mathrm{H}) ;{ }^{13} \mathrm{C} \mathrm{NMR}(100$ $\left.\mathrm{MHz}, \mathrm{CDCl}_{3}\right) \delta 198.9,162.4,159.4,154.9,148.1$, 143.9, 137.7, 137.2 , 135.0, 133.6, 132.0, 132.0, 130.8, 129.4, 129.3, 128.8, $128.6,128.3,128.1,127.5,127.4,127.1,125.4,124.4,124.1$, 108.7, 85.8, 62.7, 60.6, 60.6, 59.1, 44.0, 43.1, 21.8, 14.0, 13.9; 
HRMS(ESI) calcd for $\mathrm{C}_{43} \mathrm{H}_{40} \mathrm{NO}_{6}[\mathrm{M}+\mathrm{H}]^{+}$666.2850, found 666.2855 .

Diethyl $\quad 2$-cyclohexyl-3-(2-oxo-2-( $p$-tolyl)ethyl)-2-phenyl-2,3dihydro- $2^{\prime} H$-spiro[indene-1, $3^{\prime}$-isoxazole]- $4^{\prime}, 5^{\prime}$-dicarboxylate (5dba), $1.2: 1 \mathrm{dr}$. Purified by silica gel column chromatography ( $10: 1$ petroleum ether/ethyl acetate): $94.8 \mathrm{mg}, 78 \%$ yield; light yellow solid, mp $142-144{ }^{\circ} \mathrm{C}$, IR (film) $1746,1703,1455,1353$, 1278, $1171 \mathrm{~cm}^{-1} ;{ }^{1} \mathrm{H}$ NMR $\left(400 \mathrm{MHz}, \mathrm{CDCl}_{3}\right) \delta 7.84(\mathrm{~d}, J=$ $8.0 \mathrm{~Hz}, 1 \mathrm{H}), 7.72(\mathrm{~d}, J=8.0 \mathrm{~Hz}, 1 \mathrm{H}), 7.68-7.65(\mathrm{~m}, 1 \mathrm{H}), 7.42-7.11$ $(\mathrm{m}, 10 \mathrm{H}), 4.47(\mathrm{~d}, J=8.0 \mathrm{~Hz}, 0.4 \mathrm{H}), 4.26-3.99(\mathrm{~m}, 4 \mathrm{H}), 3.94(\mathrm{~d}, J$ $=8.0 \mathrm{~Hz}, 0.5 \mathrm{H}), 3.57(\mathrm{~d}, J=12.0 \mathrm{~Hz}, 0.4 \mathrm{H}), 3.52-3.39(\mathrm{~m}, 0.9 \mathrm{H})$, $3.29(\mathrm{dd}, J=16.0,8.0 \mathrm{~Hz}, 0.4 \mathrm{H}), 3.12(\mathrm{dd}, J=16.0,4.0 \mathrm{~Hz}, 0.4 \mathrm{H})$, $2.40(\mathrm{~s}, 1.6 \mathrm{H}), 2.38(\mathrm{~s}, 1.4 \mathrm{H}), 2.30-2.25(\mathrm{~m}, 0.5 \mathrm{H}), 2.13(\mathrm{~d}, J=$ $12.0 \mathrm{~Hz}, 0.5 \mathrm{H}), 1.97(\mathrm{~d}, J=12.0 \mathrm{~Hz}, 0.5 \mathrm{H}), 1.82-1.69(\mathrm{~m}, 1.7 \mathrm{H})$, $1.65-1.51(\mathrm{~m}, 3 \mathrm{H}), 1.31-1.18(\mathrm{~m}, 6 \mathrm{H}), 1.11(\mathrm{t}, J=8.0 \mathrm{~Hz}, 2 \mathrm{H})$, $1.02-0.86(\mathrm{~m}, 2 \mathrm{H}) ;{ }^{13} \mathrm{C}$ NMR $\left(100 \mathrm{MHz}, \mathrm{CDCl}_{3}\right) \delta$ 199.4, 199.4, 162.7 , 162.4, 159.3, 159.2, 156.3, 155.7, 148.5, 147.9, 144.2, $143.8,137.6,137.5,137.4,135.7,135.3,134.6,132.0,130.7$, 129.6, 129.4, 129.4, 129.4, 128.5, 128.2, 127.9, 127.6, 127.4, 127.3, 126.9, 126.7, 126.5, 125.7, 125.7, 124.5, 110.3, 108.8, 85.0, 83.7, 63.8, 63.7, 63.2, 62.4, 62.4, 60.7, 59.3, 44.0, 42.1, 41.8, 40.0, $33.8,32.7,29.9,27.7,27.6,26.2,25.9,25.7,25.2,25.1,21.8,21.8$, 14.2, 14.2, 14.1, 14.1, 14.0; HRMS (ESI) calcd for $\mathrm{C}_{38} \mathrm{H}_{42} \mathrm{NO}_{6}[\mathrm{M}+$ $\mathrm{H}]^{+}$608.3007, found 608.3012.

Dimethyl $\quad 2^{\prime}$-benzyl-3-(2-oxo-2-( $p$-tolyl)ethyl)-2-phenyl-2,3dihydro-2 ${ }^{\prime} H$-spiro[indene-1, $3^{\prime}$-isoxazole] $-4^{\prime}, 5^{\prime}$-dicarboxylate

(5dab), $3.2: 1 \mathrm{dr}$. Purified by silica gel column chromatography (20:1 petroleum ether/ethyl acetate): $111.7 \mathrm{mg}, 95 \%$ yield; light yellow solid, mp $123-125{ }^{\circ} \mathrm{C}$, IR (film) $1763,1754,1443$, 1352, 1296, $1142 \mathrm{~cm}^{-1}$; ${ }^{1} \mathrm{H}$ NMR (400 MHz, $\left.\mathrm{CDCl}_{3}\right) \delta 7.84(\mathrm{~d}, J=$ $8.0 \mathrm{~Hz}, 0.5 \mathrm{H}), 7.70-7.67(\mathrm{~m}, 3 \mathrm{H}), 7.47-7.40(\mathrm{~m}, 2.5 \mathrm{H}), 7.36-7.15$ $(\mathrm{m}, 10 \mathrm{H}), 7.13(\mathrm{~d}, J=4.0 \mathrm{~Hz}, 2 \mathrm{H}), 4.50(\mathrm{t}, J=8.0 \mathrm{~Hz}, 0.2 \mathrm{H}), 4.19-$ $4.10(\mathrm{~m}, 1.8 \mathrm{H}), 4.00(\mathrm{~d}, J=16.0 \mathrm{~Hz}, 0.9 \mathrm{H}), 3.91-3.81(\mathrm{~m}, 0.7 \mathrm{H})$, $3.75(\mathrm{~d}, J=4.0 \mathrm{~Hz}, 1.2 \mathrm{H}), 3.73(\mathrm{~s}, 2.2 \mathrm{H}), 3.66(\mathrm{~s}, 2.2 \mathrm{H}), 3.54-3.32$ $(\mathrm{m}, 2.7 \mathrm{H}), 3.18(\mathrm{dd}, J=4.0,16.0 \mathrm{~Hz}, 0.2 \mathrm{H}), 2.39(\mathrm{~s}, 0.7 \mathrm{H}), 2.36(\mathrm{~s}$, $2.2 \mathrm{H}) ;{ }^{13} \mathrm{C}$ NMR $\left(100 \mathrm{MHz}, \mathrm{CDCl}_{3}\right) \delta 199.1,198.8,163.3,162.9$, 159.5, 154.9, 154.4, 148.0, 147.0, 144.2, 143.9, 137.6, 137.3, 137.1, 136.9, 135.5, 135.4, 135.0, 134.6, 131.6, 130.5, 130.1, $129.7,129.4,129.3,128.9,128.6,128.5,128.3,128.2,128.0$, 127.6, 127.4, 127.3, 127.2, 126.7, 126.1, 125.8, 124.8, 109.2, 107.9, 100.2, 85.4, 84.1, 61.3, 60.7, 60.2, 58.4, 53.2, 52.1, 44.0, 42.4, 42.1, 40.5, 29.9, 21.8; HRMS (ESI) calcd for $\mathrm{C}_{37} \mathrm{H}_{34} \mathrm{NO}_{6}[\mathrm{M}+$ $\mathrm{H}]^{+}$588.2381, found 588.2379.

Ethyl $\quad 2$-benzyl-3-(2-oxo-2-( $p$-tolyl)ethyl)-2-phenyl-2,3-dihydro-2' $H$-spiro[indene-1,3'-isoxazole]-4'-carboxylate

(5dac), $1.5: 1 \mathrm{dr}$. Purified by silica gel column chromatography $(20: 1$ petroleum ether/ethyl acetate): $87.0 \mathrm{mg}, 80 \%$ yield; light yellow oil, IR (film) 1704, 1682, 1454, 1372, 1278, $1106 \mathrm{~cm}^{-1} ;{ }^{1} \mathrm{H}$ NMR $\left(400 \mathrm{MHz}, \mathrm{CDCl}_{3}\right) \delta 7.82(\mathrm{~d}, J=8.0 \mathrm{~Hz}, 1 \mathrm{H}), 7.73-7.71(\mathrm{~m}, 1 \mathrm{H})$, $7.67(\mathrm{~d}, J=8.0 \mathrm{~Hz}, 1 \mathrm{H}), 7.46-7.39(\mathrm{~m}, 2 \mathrm{H}), 7.37(\mathrm{~d}, J=4.0 \mathrm{~Hz}$, 2H), 7.34-7.27 (m, 4H), 7.26-7.21 (m, 4H), 7.19-7.12 (m, 3H), $4.45(\mathrm{t}, J=8.0 \mathrm{~Hz}, 0.6 \mathrm{H}), 4.23-4.16(\mathrm{~m}, 1.5 \mathrm{H}), 4.14-4.07(\mathrm{~m}$, $1.3 \mathrm{H}), 3.95-3.88(\mathrm{~m}, 1 \mathrm{H}), 3.73(\mathrm{~d}, J=16.0 \mathrm{~Hz}, 0.6 \mathrm{H}), 3.50-3.33$ $(\mathrm{m}, 2.4 \mathrm{H}), 3.17(\mathrm{dd}, J=16.0,4.0 \mathrm{~Hz}, 0.6 \mathrm{H}), 2.39(\mathrm{~s}, 1.8 \mathrm{H}), 2.35(\mathrm{~s}$, $1.2 \mathrm{H}), 1.26-1.23(\mathrm{~m}, 1.8 \mathrm{H}), 1.15(\mathrm{~d}, J=8.0 \mathrm{~Hz}, 1.2 \mathrm{H}),{ }^{13} \mathrm{C} \mathrm{NMR}$ $\left(100 \mathrm{MHz}, \mathrm{CDCl}_{3}\right) \delta 199.3,198.8,163.8,163.3,156.4,156.2$, $147.8,146.6,144.2,143.8,138.3,138.3,138.0,137.9,137.5$,
136.3, 135.1, 134.6, 131.6, 130.7, 129.7, 129.4, 129.4, 129.3, $128.7,128.5,128.4,128.3,128.3,128.2,127.9,127.6,127.4$, 127.3, 127.0, 126.6, 126.0, 125.7, 124.7, 108.3, 127.7, 83.3, 82.1, 61.1, 60.6, 60.6, 60.1, 57.9, 43.9, 42.4, 42.3, 40.8, 29.9, 21.8, 21.8, 14.5, 14.4; HRMS (ESI) calcd for $\mathrm{C}_{36} \mathrm{H}_{34} \mathrm{NO}_{4}[\mathrm{M}+\mathrm{H}]^{+}$544.2482, found 544.2479 .

tert-Butyl 2'-benzyl-3-(2-oxo-2-( $p$-tolyl)ethyl)-2-phenyl-2,3dihydro-2 $2^{\prime} H$-spiro[indene-1, $3^{\prime}$-isoxazole]-4'-carboxylate (5dad), $1.3: 1 \mathrm{dr}$. Purified by silica gel column chromatography $(20: 1$ petroleum ether/ethyl acetate): $85.8 \mathrm{mg}, 75 \%$ yield; reddish brown solid, mp $85-87^{\circ} \mathrm{C}$, IR (film) 1699, 1624, 1455, 1364, 1228, $1134 \mathrm{~cm}^{-1} ;{ }^{1} \mathrm{H}$ NMR $\left(500 \mathrm{MHz}, \mathrm{CDCl}_{3}\right) \delta 7.83(\mathrm{~d}, J=$ $5.0 \mathrm{~Hz}, 1 \mathrm{H}), 7.12(\mathrm{~d}, J=5.0 \mathrm{~Hz}, 1 \mathrm{H}), 7.68(\mathrm{~d}, J=10.0 \mathrm{~Hz}, 1 \mathrm{H})$, 7.47-7.36 (m, 4H), 7.34-7.21 (m, 8H), 7.18-7.11 (m, 3H), $4.43(\mathrm{t}, J$ $=10.0 \mathrm{~Hz}, 0.5 \mathrm{H}), 4.13(\mathrm{~d}, J=5.0 \mathrm{~Hz}, 0.4 \mathrm{H}), 4.10-4.05(\mathrm{~m}, 0.5 \mathrm{H})$, $3.93-3.85(\mathrm{~m}, 0.9 \mathrm{H}), 3.75(\mathrm{~d}, J=20.0 \mathrm{~Hz}, 0.5 \mathrm{H}), 3.51-3.37(\mathrm{~m}$, $1.8 \mathrm{H}), 3.31(\mathrm{dd}, J=20.0,10.0 \mathrm{~Hz}, 0.6 \mathrm{H}), 3.17(\mathrm{dd}, J=15.0$, $5.0 \mathrm{~Hz}, 0.5 \mathrm{H}), 2.40(\mathrm{~s}, 1.7 \mathrm{H}), 2.36(\mathrm{~s}, 1.3 \mathrm{H}), 1.40(\mathrm{~s}, 5 \mathrm{H}), 1.30(\mathrm{~s}$, $4 \mathrm{H}) ;{ }^{13} \mathrm{C}$ NMR $\left(125 \mathrm{MHz}, \mathrm{CDCl}_{3}\right) \delta 199.3,198.9,163.3,162.8$, $156.0,155.9,147.9,146.6,144.2$, 143.8, 138.6, 138.6, 138.1, $138.0,137.7,136.4,135.1,134.6,131.7,130.7,129.6,129.4$, $129.4,129.3,129.2,128.6,128.5,128.4,128.3,128.2,128.2$, $127.9,127.5,127.4,127.2,127.2,126.9,126.9,126.5,126.2$, 125.8, 124.6, 109.9, 109.1, 83.3, 82.1, 80.6, 80.5, 61.1, 60.9, 60.7, 58.0, 43.9, 42.5, 42.4, 40.7, 28.4, 28.2, 21.8, 21.8; HRMS (ESI) calcd for $\mathrm{C}_{38} \mathrm{H}_{37} \mathrm{NO}_{6} \mathrm{Na}[\mathrm{M}+\mathrm{Na}]^{+}$594.2615, found 594.2615.

2-(4'-Acetyl-2'-benzyl-2-phenyl-2,3-dihydro-2' $H$-spiro[indene1,3'-isoxazol]-3-yl)-1-( $p$-tolyl)ethan-1-one (5dae), $4.0: 1 \mathrm{dr}$. Purified by silica gel column chromatography $(30: 1$ petroleum ether/ethyl acetate): $98.6 \mathrm{mg}$, 96\% yield; white solid, mp 71$73{ }^{\circ} \mathrm{C}$, IR (film) 1679, 1653, 1457, 1374, 1228, $1140 \mathrm{~cm}^{-1} ;{ }^{1} \mathrm{H}$ $\operatorname{NMR}\left(400 \mathrm{MHz}, \mathrm{CDCl}_{3}\right) \delta 7.82(\mathrm{~d}, J=8.0 \mathrm{~Hz}, 1.5 \mathrm{H}), 7.70-7.65(\mathrm{~m}$, $0.6 \mathrm{H}), 7.45-7.34(\mathrm{~m}, 3 \mathrm{H}), 7.31-7.17(\mathrm{~m}, 12 \mathrm{H}), 7.13(\mathrm{~d}, J=4.0 \mathrm{~Hz}$, $1 \mathrm{H}), 4.44(\mathrm{~d}, J=8.0 \mathrm{~Hz}, 0.8 \mathrm{H}), 4.20(\mathrm{~d}, J=8.0 \mathrm{~Hz}, 0.2 \mathrm{H}), 4.13-$ $4.07(\mathrm{~m}, 0.2 \mathrm{H}), 4.03(\mathrm{~d}, J=12.0 \mathrm{~Hz}, 0.8 \mathrm{H}), 3.86(\mathrm{~d}, J=16.0 \mathrm{~Hz}$, $0.2 \mathrm{H}), 3.70(\mathrm{~d}, J=16.0 \mathrm{~Hz}, 0.8 \mathrm{H}), 3.48-3.36(\mathrm{~m}, 2 \mathrm{H}), 3.13(\mathrm{dd}, J=$ 16.0, $4.0 \mathrm{~Hz}, 0.8 \mathrm{H}), 2.38(\mathrm{~s}, 2.4 \mathrm{H}), 2.35(\mathrm{~s}, 0.6 \mathrm{H}), 2.32(\mathrm{~s}, 2.3 \mathrm{H})$, $2.22(\mathrm{~s}, 0.5 \mathrm{H}) ;{ }^{13} \mathrm{C}$ NMR $\left(100 \mathrm{MHz}, \mathrm{CDCl}_{3}\right) \delta 199.4,198.8,191.7$, 191.3, 157.7, 157.6, 147.6, 146.5, 144.1, 143.8, 138.2, 137.9, $137.9,137.8,137.4,136.5,135.0,134.6,131.6,130.7,129.6$, $129.4,129.3,128.7,128.5,128.3,128.2,128.2,127.9,127.6$, $127.3,127.3,127.2,127.0,126.7,125.9,125.4,124.8$, 118.1, 117.9, 83.6, 82.3, 61.2, 60.7, 59.8, 57.2, 43.9, 42.4, 42.2, 40.8, 29.9, 28.1, 28.0, 21.8, 21.8; HRMS (ESI) calcd for $\mathrm{C}_{35} \mathrm{H}_{32} \mathrm{NO}_{3}[\mathrm{M}+$ $\mathrm{H}]^{+}$514.2377, found 514.2380.

Methyl 2'-benzyl-3-(2-oxo-2-( $p$-tolyl)ethyl)-2,5'-diphenyl-2,3dihydro-2 ${ }^{\prime} H$-spiro-[indene-1, $3^{\prime}$-isoxazole]- $4^{\prime}$-carboxylate (5daf), $2.2: 1 \mathrm{dr}$. Purified by silica gel column chromatography $(10: 1$ petroleum ether/ethyl acetate): $93.2 \mathrm{mg}, 77 \%$ yield; yellow white solid, mp 73-75 ${ }^{\circ} \mathrm{C}$, IR (film) 1738, 1697, 1495, 1350, 1241, $1092 \mathrm{~cm}^{-1} ;{ }^{1} \mathrm{H}$ NMR $\left(400 \mathrm{MHz}, \mathrm{CDCl}_{3}\right) \delta 7.85(\mathrm{~d}, J=8.0 \mathrm{~Hz}$, $1.5 \mathrm{H}), 7.74(\mathrm{t}, J=8.0 \mathrm{~Hz}, 1 \mathrm{H}), 7.53(\mathrm{~d}, J=4.0 \mathrm{~Hz}, 1.5 \mathrm{H}), 7.43-$ $7.13(\mathrm{~m}, 19 \mathrm{H}), 4.59(\mathrm{t}, J=8.0 \mathrm{~Hz}, 0.7 \mathrm{H}), 4.25(\mathrm{~d}, J=8.0 \mathrm{~Hz}$, $0.3 \mathrm{H}), 4.17-4.12(\mathrm{~m}, 0.3 \mathrm{H}), 4.05(\mathrm{~d}, J=16.0 \mathrm{~Hz}, 0.3 \mathrm{H}), 3.93-3.85$ $(\mathrm{m}, 1.3 \mathrm{H}), 3.59(\mathrm{~s}, 2.7 \mathrm{H}), 3.55-3.45(\mathrm{~m}, 1.5 \mathrm{H}), 3.41-3.34(\mathrm{~m}$, $0.8 \mathrm{H}), 3.22-3.17(\mathrm{~m}, 0.8 \mathrm{H}), 2.39(\mathrm{~s}, 2.1 \mathrm{H}), 2.37(\mathrm{~s}, 0.9 \mathrm{H}) ;{ }^{13} \mathrm{C}$ NMR $\left(100 \mathrm{MHz}, \mathrm{CDCl}_{3}\right) \delta$ 199.4, 199.2, 166.7, 164.9, 148.1, 147.1, 
144.1, 143.8, 138.6, 138.1, 138.0, 137.8, 136.4, 134.7, 131.9, $130.6,130.5$, 129.6, 129.4, 129.4, 129.3, 129.2, 128.6, 128.6, $128.5,128.4,128.3,128.3,128.2$, 128.0, 127.9, 127.9, 127.8, $127.4,127.3,127.0,127.0,126.8,126.7,126.2$, 125.8, 124.7, 101.7, 86.1, 84.8, 62.2, 60.5, 60.2, 58.8, 51.2, 3.9, 42.9, 42.2, 40.5, 29.9, 21.8, 21.8; HRMS (ESI) calcd for $\mathrm{C}_{41} \mathrm{H}_{36} \mathrm{NO}_{4}[\mathrm{M}+\mathrm{H}]^{+}$ 606.2639, found 606.2646.

Ethyl 2'-benzyl-3-(2-oxo-2-( $p$-tolyl)ethyl)-2,5'-diphenyl-2,3dihydro-2 ${ }^{\prime} H$-spiro[indene-1, $3^{\prime}$-isoxazole]- $4^{\prime}$-carboxylate (5dag), $1.5: 1 \mathrm{dr}$. Purified by silica gel column chromatography $10: 1$ petroleum ether/ethyl acetate): $99.2 \mathrm{mg}, 80 \%$ yield; yellow white solid, mp 81-83 ${ }^{\circ} \mathrm{C}$, IR (film) 1695, 1646, 1454, 1372, 1336, $1091 \mathrm{~cm}^{-1}$; ${ }^{1} \mathrm{H}$ NMR $\left(400 \mathrm{MHz}, \mathrm{CDCl}_{3}\right) \delta 7.85(\mathrm{~d}, J=8.0 \mathrm{~Hz}$, $1.3 \mathrm{H}), 7.74(\mathrm{t}, J=8.0 \mathrm{~Hz}, 1.4 \mathrm{H}), 7.53(\mathrm{~d}, J=8.0 \mathrm{~Hz}, 1.4 \mathrm{H}), 7.44-$ $7.13(\mathrm{~m}, 19 \mathrm{H}), 4.58(\mathrm{t}, J=8.0 \mathrm{~Hz}, 0.6 \mathrm{H}), 4.26(\mathrm{~d}, J=8.0 \mathrm{~Hz}$, $0.4 \mathrm{H}), 4.17-3.96(\mathrm{~m}, 2.3 \mathrm{H}), 3.94-3.86(\mathrm{~m}, 1.5 \mathrm{H}), 3.66-3.59(\mathrm{~m}$, $1.1 \mathrm{H}), 3.53(\mathrm{~d}, J=12.0 \mathrm{~Hz}, 0.7 \mathrm{H}), 3.35$ (dd, $J=20.0,8.0 \mathrm{~Hz}$, $0.7 \mathrm{H}), 3.20(\mathrm{dd}, J=16.0,4.0 \mathrm{~Hz}, 0.6 \mathrm{H}), 2.40(\mathrm{~s}, 1.8 \mathrm{H}), 2.37$ (s, $1.2 \mathrm{H}), 0.99(\mathrm{~d}, J=8.0 \mathrm{~Hz}, 1.8 \mathrm{H}), 0.90(\mathrm{~d}, J=8.0 \mathrm{~Hz}, 1.2 \mathrm{H}) ;{ }^{13} \mathrm{C}$ NMR (100 MHz, $\left.\mathrm{CDCl}_{3}\right) \delta 199.4,199.2,166.5,166.4,166.0,148.2$, $147.1,144.1,143.8,141.3,138.9,138.8,138.2$, 138.0, 137.9, $136.5,135.2$, 134.7, 131.9, 130.6, 130.6, 129.5, 129.4, 129.4, $129.3,129.2$, 128.6, 128.6, 128.5, 128.4, 128.3, 128.3, 128.2, $128.0,127.9,127.8,127.8,127.4,127.2,127.0,126.9,126.8$, 126.6, 126.3, 125.9, 124.6, 102.7, 102.0, 86.0, 84.8, 62.2, 60.5, 60.2, 60.0, 59.9, 58.8, 43.9, 42.9, 42.2, 40.6, 32.1, 29.9, 21.8, 21.8, 14.0, 13.9; HRMS (ESI) calcd for $\mathrm{C}_{42} \mathrm{H}_{37} \mathrm{NO}_{4} \mathrm{Na}[\mathrm{M}+\mathrm{Na}]^{+}$ 642.2615, found 642.2610.

2-(4'-Acetyl-2'-benzyl-2,5'-diphenyl-2,3-dihydro-2' $H$-spiro [indene-1,3'-isoxazol]-3-yl)-1-( $p$-tolyl)ethan-1-one (5dah), $5.0: 1$ dr. Purified by silica gel column chromatography $(20: 1$ petroleum ether/ethyl acetate): $96.7 \mathrm{mg}, 82 \%$ yield; light yellow solid, mp 70-72 ${ }^{\circ} \mathrm{C}$, IR (film) 1736, 1680, 1454, 1371, 1241, $1118 \mathrm{~cm}^{-1}$; ${ }^{1} \mathrm{H}$ NMR $\left(400 \mathrm{MHz}, \mathrm{CDCl}_{3}\right) \delta 7.85(\mathrm{~d}, J=8.0 \mathrm{~Hz}, 1.5 \mathrm{H}), 7.75-7.72$ $(\mathrm{m}, 0.6 \mathrm{H}), 7.53(\mathrm{~d}, J=8.0 \mathrm{~Hz}, 1.5 \mathrm{H}), 7.42-7.13(\mathrm{~m}, 19 \mathrm{H}), 6.97$ (d, $J=8.0 \mathrm{~Hz}, 0.6 \mathrm{H}), 4.59(\mathrm{t}, J=8.0 \mathrm{~Hz}, 0.8 \mathrm{H}), 4.32-4.26(\mathrm{~m}, 0.2 \mathrm{H})$, $4.17-4.09(\mathrm{~m}, 0.2 \mathrm{H}), 4.05(\mathrm{~d}, J=12.0 \mathrm{~Hz}, 0.1 \mathrm{H}), 3.95-3.88(\mathrm{~m}$, $1.7 \mathrm{H}), 3.64(\mathrm{t}, J=8.0 \mathrm{~Hz}, 0.5 \mathrm{H}), 3.52-3.39(\mathrm{~m}, 1.8 \mathrm{H}), 3.16(\mathrm{dd}, J=$ 16.0, 4.0 Hz,1H), 2.39 (s, 2.4H), 2.37 (s, 0.6H), $1.97(\mathrm{~s}, 2.5 \mathrm{H}), 1.83$ $(\mathrm{s}, 0.5 \mathrm{H}) ;{ }^{13} \mathrm{C}$ NMR $\left(100 \mathrm{MHz}, \mathrm{CDCl}_{3}\right) \delta 199.5,199.2,194.0$, $167.9,148.2$, 147.1, 144.1, 143.7, 138.3, 138.1, 137.8, 136.6, $135.3,134.7,132.0,131.0,130.9,130.7,129.5,129.4,129.4$, $129.3,129.2$, 128.9, 128.8, 128.7, 128.6, 128.5, 128.3, 128.0, $127.8,127.5,127.3,127.1,126.9,126.8,126.2,125.7,124.8$, 113.4, 85.5, 61.7, 60.3, 58.5, 43.8, 43.0, 42.1, 40.7, 30.2, 30.0, 29.9, 21.8; HRMS (ESI) calcd for $\mathrm{C}_{41} \mathrm{H}_{36} \mathrm{NO}_{3}[\mathrm{M}+\mathrm{H}]^{+} 590.2690$, found 590.2692 .

2-(2'-Benzyl-4'-(2,4-dinitrophenyl)-2,5'-diphenyl-2,3-dihydro$2^{\prime} H$-spiro[indene-1,3'-isoxazol]-3-yl)-1-( $p$-tolyl)ethan-1-one

(5dai), $1.8: 1 \mathrm{dr}$. Purified by silica gel column chromatography (20:1 petroleum ether/ethyl acetate): $121.3 \mathrm{mg}, 85 \%$ yield; brownish red solid, mp $135-137^{\circ} \mathrm{C}$, IR (film) 1721, 1680, 1536, $1453,1345,1180 \mathrm{~cm}^{-1} ;{ }^{1} \mathrm{H}$ NMR $\left(400 \mathrm{MHz}, \mathrm{CDCl}_{3}\right) \delta 8.42(\mathrm{~s}, 1 \mathrm{H})$, $8.31(\mathrm{~d}, J=8.0 \mathrm{~Hz}, 1 \mathrm{H}), 7.87(\mathrm{~d}, J=8.0 \mathrm{~Hz}, 2 \mathrm{H}), 7.53-7.14(\mathrm{~m}$, $20 \mathrm{H}), 6.97(\mathrm{~d}, J=8.0 \mathrm{~Hz}, 2 \mathrm{H}), 4.70(\mathrm{~s}, 0.7 \mathrm{H}), 4.25-4.03(\mathrm{~m}, 1.6 \mathrm{H})$, $3.90(\mathrm{~d}, J=8.0 \mathrm{~Hz}, 1 \mathrm{H}), 3.63(\mathrm{~d}, J=12.0 \mathrm{~Hz}, 1 \mathrm{H}), 3.41(\mathrm{~d}, J=$ $4.0 \mathrm{~Hz}, 1.7 \mathrm{H}), 2.42(\mathrm{~s}, 1.9 \mathrm{H}), 2.37(\mathrm{~s}, 1.1 \mathrm{H}), 3.71(\mathrm{~d}, J=16.0 \mathrm{~Hz}$,
$0.6 \mathrm{H}), 3.49-3.28(\mathrm{~m}, 2.4 \mathrm{H}), 3.17(\mathrm{dd}, J=16.0,4.0 \mathrm{~Hz}, 0.5 \mathrm{H}), 2.39$ $(\mathrm{s}, 1.6 \mathrm{H}), 2.35(\mathrm{~s}, 1.4 \mathrm{H}), 2.06(\mathrm{~s}, 1.5 \mathrm{H}), 2.00(\mathrm{~s}, 1.4 \mathrm{H}), 1.14(\mathrm{t}, J=$ $8.0 \mathrm{~Hz}, 1.6 \mathrm{H}), 1.05$ (d, $J=8.0 \mathrm{~Hz}, 1.4 \mathrm{H}) ;{ }^{13} \mathrm{C} \mathrm{NMR}(100 \mathrm{MHz}$, $\left.\mathrm{CDCl}_{3}\right) \delta 198.7,150.5,145.5,144.4,144.0,138.1,137.8,135.7$, 135.6 , 135.4, 134.7, 131.1, 131.0, 130.1, 129.5, 129.3, 129.0, $128.8,128.7,128.6,128.5,128.4,128.1,128.0$, 127.8, 127.6, 127.4, 127.2, 126.8, 126.6, 124.6, 121.0, 117.9, 88.8, 86.9, 59.6, 41.3, 34.6, 29.8, 21.9; HRMS (ESI) calcd for $\mathrm{C}_{45} \mathrm{H}_{35} \mathrm{~N}_{3} \mathrm{O}_{6} \mathrm{Na}[\mathrm{M}+$ $\mathrm{Na}]^{+} 736.2418$, found 736.2425 .

Ethyl 2'-benzyl-5'-methyl-3-(2-oxo-2-( $p$-tolyl)ethyl)-2-phenyl2,3-dihydro-2 ${ }^{\prime} H$-spiro[indene-1,3'-isoxazole]-4' ${ }^{\prime}$-carboxylate

(5daj), 1.1 : 1 dr. Purified by silica gel column chromatography (20:1 petroleum ether/ethyl acetate): $50.2 \mathrm{mg}, 45 \%$ yield; brown solid, mp 39-41 ${ }^{\circ} \mathrm{C}$, IR (film) 1734, 1696, 1473, 1374, 1258, $1107 \mathrm{~cm}^{-1}$; ${ }^{1} \mathrm{H}$ NMR (400 $\left.\mathrm{MHz}, \mathrm{CDCl}_{3}\right) \delta 7.83(\mathrm{~d}, J=$ $8.0 \mathrm{~Hz}, 1 \mathrm{H}), 7.69(\mathrm{t}, J=8.0 \mathrm{~Hz}, 2 \mathrm{H}), 7.41-7.33(\mathrm{~m}, 4 \mathrm{H}), 7.31-7.09$ $(\mathrm{m}, 11 \mathrm{H}), 4.48(\mathrm{t}, J=8.0 \mathrm{~Hz}, 0.5 \mathrm{H}), 4.23-3.98(\mathrm{~m}, 3 \mathrm{H}), 3.87-3.78$ (m, $1 \mathrm{H}), 3.71(\mathrm{~d}, J=16.0 \mathrm{~Hz}, 0.6 \mathrm{H}), 3.49-3.28(\mathrm{~m}, 2.4 \mathrm{H}), 3.17$ (dd, $J=16.0,4.0 \mathrm{~Hz}, 0.5 \mathrm{H}), 2.39(\mathrm{~s}, 1.6 \mathrm{H}), 2.35(\mathrm{~s}, 1.4 \mathrm{H}), 2.06(\mathrm{~s}$, $1.5 \mathrm{H}), 2.00(\mathrm{~s}, 1.4 \mathrm{H}), 1.14(\mathrm{t}, J=8.0 \mathrm{~Hz}, 1.6 \mathrm{H}), 1.05(\mathrm{~d}, J=8.0 \mathrm{~Hz}$, $1.4 \mathrm{H}) ;{ }^{13} \mathrm{C}$ NMR $\left(100 \mathrm{MHz}, \mathrm{CDCl}_{3}\right) \delta 199.4,199.1,168.5,168.3$, $164.9,164.6,147.7,146.7,144.1,143.7,139.4,139.1,138.3$, $137.9,136.6,135.1,134.7,131.6,130.5,129.4,129.3,129.3$, 129.0 , 128.6, 128.5, 128.5, 128.3, 128.2, 128.1, 127.7, 127.4, $127.3,127.0,126.7,126.7,126.4,126.2$, 125.9, 124.5, 102.1, 101.6, 84.7, 83.5, 61.4, 60.8, 60.3, 59.7, 59.6, 58.1, 43.9, 42.6, 42.3, 40.3, 29.9, 21.8, 21.8, 14.4, 14.3, 13.2, 13.1; HRMS (ESI) calcd for $\mathrm{C}_{37} \mathrm{H}_{35} \mathrm{NO}_{4} \mathrm{Na}[\mathrm{M}+\mathrm{Na}]^{+} 580.2458$, found 580.2460.

2'-Benzyl-5'-methyl-3-(2-oxo-2-( $p$-tolyl)ethyl)-2-phenyl2,3,3a' $6 \mathrm{a}^{\prime}$-tetrahydro-2' $H, 4^{\prime} H$-spiro[indene-1,3'-pyrrolo[3,4-d] isoxazole]-4 $4^{\prime}, 6^{\prime}\left(5^{\prime} H\right)$-dione (5dak), $2.0: 1 \mathrm{dr}$. Purified by silica gel column chromatography (10:1 petroleum ether/ethyl acetate): $76.8 \mathrm{mg}$, 69\% yield; yellowish white solid, mp 110-112 ${ }^{\circ} \mathrm{C}$, IR (film) 1709, 1677, 1453, 1377, 1281, $1181 \mathrm{~cm}^{-1} ;{ }^{1} \mathrm{H}$ NMR (400 $\left.\mathrm{MHz} \mathrm{CDCl}_{3}\right) \delta 7.80(\mathrm{~d}, J=8.0 \mathrm{~Hz}, 0.7 \mathrm{H}), 7.54(\mathrm{~d}, J=8.0 \mathrm{~Hz}$, $1.2 \mathrm{H}), 7.41-7.17(\mathrm{~m}, 15 \mathrm{H}), 7.10(\mathrm{~d}, J=4.0 \mathrm{~Hz}, 0.8 \mathrm{H}), 7.00(\mathrm{~d}, J=$ $8.0 \mathrm{~Hz}, 0.5 \mathrm{H}), 4.94$ (d, $J=8.0 \mathrm{~Hz}, 0.6 \mathrm{H}), 4.66$ (d, $J=8.0 \mathrm{~Hz}$, $0.3 \mathrm{H}), 4.56-4.51(\mathrm{~m}, 0.1 \mathrm{H}), 4.37-4.29(\mathrm{~m}, 0.5 \mathrm{H}), 4.11-3.95(\mathrm{~m}$, $2.1 \mathrm{H}), 3.90-3.78(\mathrm{~m}, 1 \mathrm{H}), 3.73-3.67(\mathrm{~m}, 0.4 \mathrm{H}), 3.51-3.44(\mathrm{~m}$, $0.4 \mathrm{H}), 3.36-3.29(\mathrm{~m}, 1.2 \mathrm{H}), 3.21-3.15(\mathrm{~m}, 1.3 \mathrm{H}), 3.00(\mathrm{~s}, 1 \mathrm{H})$, $2.50(\mathrm{~s}, 2 \mathrm{H}), 2.39(\mathrm{~s}, 1 \mathrm{H}), 2.38(\mathrm{~s}, 2 \mathrm{H}) ;{ }^{13} \mathrm{C} \mathrm{NMR}\left(100 \mathrm{MHz}, \mathrm{CDCl}_{3}\right)$ $\delta$ 198.8, 175.2, 173.9, 172.7, 146.5, 145.0, 144.3, 141.3, 137.4, 137.2 , 136.8, 134.9, 134.7, 134.4, 134.4, 132.1, 130.7, 129.8, $129.6,129.5$, 128.8, 128.5, 128.4, 128.4, 128.3, 128.2, 128.2, $128.1,128.0,127.7,127.6,127.2$, 127.1, 125.3, 124.9, 124.7, 124.7, 79.8, 78.8, 75.4, 60.3, 58.7, 58.1, 56.8, 56.4, 56.0, 46.5, 43.4, 43.0, 42.6, 29.9, 24.9, 24.4, 21.8. HRMS (ESI) calcd for $\mathrm{C}_{36} \mathrm{H}_{32} \mathrm{~N}_{2} \mathrm{O}_{4} \mathrm{Na}[\mathrm{M}+\mathrm{Na}]^{+} 579.2254$, found 579.2259 .

Representative procedure for the cycloaddition reaction of generated in situ ketonitrone (Table 3, 5aaa). A $10 \mathrm{~mL}$ roundbottom flask was charged with $1 \mathrm{a}(0.1542 \mathrm{~g}, 0.50 \mathrm{mmol}), 2 \mathrm{a}$ (0.0798 g, $0.50 \mathrm{mmol})$, EtONa (0.0443 g, $0.65 \mathrm{mmol})$, and DCM $(5.0 \mathrm{~mL})$ under air atmosphere. The reaction mixture was stirred at room temperature for $12 \mathrm{~h}$, then filtered through a short pad of silica gel, and diethyl acetylenedicarboxylate $(0.1277 \mathrm{~g}, 0.75$ $\mathrm{mmol}$ ) was added. The reaction was allowed to stir under argon at $40{ }^{\circ} \mathrm{C}$ for $23 \mathrm{~h}$, and then was concentrated. The crude residue 
was purified by flash chromatography on silica gel (petroleum ether/ethyl acetate from $50: 1$ to $10: 1$ ) to afford the desired product 5aaa as a light yellow solid $(0.2286 \mathrm{~g}$, 76\% yield, $9: 1$ dr).

Diethyl 2'-benzyl-3-(2-oxo-2-phenylethyl)-2-phenyl-2,3-dihydro-2' $H$-spiro[indene-1,3'-isoxazole]-4' ${ }^{\prime}, 5^{\prime}$-dicarboxylate (5aaa), $9: 1 \mathrm{dr}$. Purified by silica gel column chromatography (petroleum ether/ethyl acetate from $50: 1$ to $10: 1$ ): $228.6 \mathrm{mg}, 76 \%$ yield; light yellow solid, mp 127-129 ${ }^{\circ} \mathrm{C}$, IR (film) 1740, 1706, 1454, 1393, 1239, $1106 \mathrm{~cm}^{-1} ;{ }^{1} \mathrm{H}$ NMR (400 MHz, $\left.\mathrm{CDCl}_{3}\right) \delta 7.89$ $(\mathrm{d}, J=8.0 \mathrm{~Hz}, 2 \mathrm{H}), 7.67(\mathrm{~d}, J=8.0 \mathrm{~Hz}, 2 \mathrm{H}), 7.52-7.42(\mathrm{~m}, 3 \mathrm{H})$, 7.39-7.20 (m, 12H), 4.21-4.08 (m, 6H), 4.02 (d, $J=16.0 \mathrm{~Hz}, 1 \mathrm{H})$, 3.56-3.42 (m, 3H), 1.31-1.19 (m, 3.3H), $1.13(\mathrm{t}, J=8.0 \mathrm{~Hz}, 2.7 \mathrm{H})$; ${ }^{13} \mathrm{C}$ NMR $\left(100 \mathrm{MHz}, \mathrm{CDCl}_{3}\right) \delta 199.2,162.4,159.2,154.8,148.0$, $137.8,137.5$, 137.4, 137.2, 133.1, 131.6, 129.7, 129.0, 128.7, 128.6, 128.2, 128.1, 127.6, 127.3, 127.2, 126.6, 126.3, 108.7, 85.3, 62.6, 60.9, 60.6, 58.4, 43.9, 42.6, 14.1, 14.0.

Diethyl 2'-benzyl-3-(2-oxo-2-(o-tolyl)ethyl)-2-phenyl-2,3-dihydro-2 ${ }^{\prime} H$-spiro[indene-1, $3^{\prime}$-isoxazole $]-4^{\prime}, 5^{\prime}$-dicarboxylate (5baa), 23.0:1 dr. Purified by silica gel column chromatography (petroleum ether/ethyl acetate from $50: 1$ to $10: 1$ ): $200.1 \mathrm{mg}$, $65 \%$ yield; light yellow solid, mp 96-97 ${ }^{\circ} \mathrm{C}$, IR (film) 1743, 1719, 1495, 1393, 1300, $1176 \mathrm{~cm}^{-1} ;{ }^{1} \mathrm{H}$ NMR (400 MHz, $\left.\mathrm{CDCl}_{3}\right) \delta 7.64-$ $7.62(\mathrm{~m}, 2 \mathrm{H}), 7.46-7.43(\mathrm{~m}, 2 \mathrm{H}), 7.39$ (d, $J=8.0 \mathrm{~Hz}, 1 \mathrm{H}), 7.34-$ 7.31 (m, 3H), 7.29-7.18 (m, 9H), 7.16-7.12 (m, 1H), 4.19-4.08 $(\mathrm{m}, 6 \mathrm{H}), 3.99(\mathrm{~d}, J=12.0 \mathrm{~Hz}, 1 \mathrm{H}), 3.52(\mathrm{~d}, J=12.0 \mathrm{~Hz}, 1 \mathrm{H}), 3.48-$ $3.41(\mathrm{~m}, 2 \mathrm{H}), 2.49(\mathrm{~s}, 0.1 \mathrm{H}), 2.36(\mathrm{~s}, 3 \mathrm{H}), 1.22(\mathrm{t}, J=8.0 \mathrm{~Hz}, 3 \mathrm{H})$, $1.13(\mathrm{t}, J=8.0 \mathrm{~Hz}, 3 \mathrm{H}) ;{ }^{13} \mathrm{C} \mathrm{NMR}\left(100 \mathrm{MHz}, \mathrm{CDCl}_{3}\right) \delta 202.8$, $162.4,159.2$, 154.8, 148.2, 138.4, 138.3, 137.8, 137.3, 137.2, 132.1 , 131.7, 131.4, 129.7, 129.0, 128.7, 128.5, 128.2, 127.6, 127.4, 127.2, 126.4, 126.3, 125.7, 108.7, 85.3, 62.6, 60.9, 60.6, 58.4, 45.6, 43.9, 21.4, 14.1, 14.0 .

Diethyl 2'-benzyl-3-(2-oxo-2-( $p$-tolyl)ethyl)-2-phenyl-2,3-dihydro-2 ${ }^{\prime} H$-spiro[indene-1, $3^{\prime}$-isoxazole $]-4^{\prime}, 5^{\prime}$-dicarboxylate (5daa), 14.0: $1 \mathrm{dr}$. Purified by silica gel column chromatography (petroleum ether/ethyl acetate from $50: 1$ to $10: 1$ ): $243.2 \mathrm{mg}$, 79\% yield; $112.1 \mathrm{mg}, 89 \%$ yield; light yellow solid, mp 140$142{ }^{\circ} \mathrm{C}$, IR (film) 1740, 1713, 1474, 1370, 1241, $1140 \mathrm{~cm}^{-1} ;{ }^{1} \mathrm{H}$ NMR (400 MHz, $\left.\mathrm{CDCl}_{3}\right) \delta 7.68(\mathrm{~d}, J=8.0 \mathrm{~Hz}, 4 \mathrm{H}), 7.43-7.39(\mathrm{~m}$, 2H), 7.32-7.15 (m, 12H), 4.21-4.10 (m, 6H), 4.02 (d, $J=12.0 \mathrm{~Hz}$, $1 \mathrm{H}), 3.54-3.39(\mathrm{~m}, 3 \mathrm{H}), 2.40(\mathrm{~s}, 0.2 \mathrm{H}), 2.37(\mathrm{~s}, 2.8 \mathrm{H}), 1.23(\mathrm{t}, J=$ $8.0 \mathrm{~Hz}, 3.2 \mathrm{H}), 1.13(\mathrm{t}, J=8.0 \mathrm{~Hz}, 2.8 \mathrm{H}) ;{ }^{13} \mathrm{C} \mathrm{NMR}(100 \mathrm{MHz}$, $\left.\mathrm{CDCl}_{3}\right) \delta 198.8,162.4,159.2,154.8,148.1,143.9,137.8,137.5$, 137.2 , 135.1, 131.6, 129.7, 129.4, 129.0, 128.6, 128.3, 128.2, 127.6, 127.3, 127.1, 126.6, 126.3, 108.8, 85.3, 62.6, 60.9, 60.6, 58.4, 44.0, 42.5, 21.8, 14.1, 14.0 .

Diethyl 2'-benzyl-3-(2-(4-bromophenyl)-2-oxoethyl)-2-phenyl2,3-dihydro-2 ${ }^{\prime} H$-spiro[indene-1,3'-isoxazole]-4' ${ }^{\prime}, 5^{\prime}$-dicarboxylate (5eaa), $5.5: 1 \mathrm{dr}$. Purified by silica gel column chromatography (petroleum ether/ethyl acetate from $50: 1$ to $10: 1$ ): $251.8 \mathrm{mg}$, 74\% yield; light yellow solid, mp115-117 ${ }^{\circ} \mathrm{C}$, IR (film) 1735, 1707, 1499, 1370, 1242, $1169 \mathrm{~cm}^{-1}$; ${ }^{1} \mathrm{H}$ NMR (400 MHz, $\mathrm{CDCl}_{3}$ ) $\delta 7.77(\mathrm{~d}, J=8.0 \mathrm{~Hz}, 0.4 \mathrm{H}), 7.66-7.56(\mathrm{~m}, 3.5 \mathrm{H}), 7.50-7.37(\mathrm{~m}$, $4 \mathrm{H}), 7.35-7.14(\mathrm{~m}, 10 \mathrm{H}), 4.22-4.08(\mathrm{~m}, 5.2 \mathrm{H}), 4.03(\mathrm{~d}, J=$ $12.0 \mathrm{~Hz}, 0.9 \mathrm{H}), 3.89-3.76(\mathrm{~m}, 0.4 \mathrm{H}), 3.54-3.48(\mathrm{~m}, 1.8 \mathrm{H}), 3.38-$ $3.28(\mathrm{~m}, 1 \mathrm{H}), 3.20-3.14(\mathrm{~m}, 0.2 \mathrm{H}), 1.29-1.20(\mathrm{~m}, 3.6 \mathrm{H}), 1.13(\mathrm{t}, J$ $=8.0 \mathrm{~Hz}, 2.5 \mathrm{H}) ;{ }^{13} \mathrm{C} \mathrm{NMR}\left(100 \mathrm{MHz}, \mathrm{CDCl}_{3}\right) \delta$ 198.1, 162.4,
159.2, 154.8, 147.7, 137.8, 137.4, 137.1, 136.1, 132.1, 131.9, $131.5,130.5,129.9,129.7,129.7,128.9,128.6,128.6,128.3$, 128.2, 128.0, 127.7, 127.4, 127.3, 126.4, 126.4, 108.6, 85.3, 62.7, 60.9, 60.6, 58.3, 43.9, 42.4, 14.1, 14.0 .

Diethyl 2'-benzyl-3-(2-(furan-2-yl)-2-oxoethyl)-2-phenyl-2,3dihydro-2' $H$-spiro[indene-1, $3^{\prime}$-isoxazole]- $4^{\prime}, 5^{\prime}$-dicarboxylate

(5faa), $33.0: 1 \mathrm{dr}$. Purified by silica gel column chromatography (petroleum ether/ethyl acetate from $50: 1$ to $10: 1$ ): $210.1 \mathrm{mg}$, 71\% yield; light yellow solid, mp 141-143 ${ }^{\circ} \mathrm{C}$, IR (film) 1739, 1710, 1465, 1371, 1189, $1140 \mathrm{~cm}^{-1}$; ${ }^{1} \mathrm{H}$ NMR (500 MHz, $\mathrm{CDCl}_{3}$ ) $\delta 7.68(\mathrm{~d}, J=10.0 \mathrm{~Hz}, 2 \mathrm{H}), 7.45-7.42(\mathrm{~m}, 2 \mathrm{H}), 7.40(\mathrm{~d}, J=5.0 \mathrm{~Hz}$, $2 \mathrm{H}), 7.36-7.33(\mathrm{~m}, 3 \mathrm{H}), 7.30-7.20(\mathrm{~m}, 6 \mathrm{H}), 7.00(\mathrm{~d}, J=5.0 \mathrm{~Hz}$, $1 \mathrm{H}), 6.50-6.49(\mathrm{~m}, 0.03 \mathrm{H}), 6.44-6.43(\mathrm{~m}, 1 \mathrm{H}), 4.20-4.15(\mathrm{~m}, 3 \mathrm{H})$, $4.14-4.06$ (m, 3H), 4.01 (d, $J=15.0 \mathrm{~Hz}, 1 \mathrm{H}), 3.54(\mathrm{~d}, J=15.0 \mathrm{~Hz}$, $1 \mathrm{H}), 3.47$ (dd, $J=20.0,10.0 \mathrm{~Hz}, 1 \mathrm{H}), 3.21$ (dd, $J=20.0,5.0 \mathrm{~Hz}$, $1 \mathrm{H}), 1.23(\mathrm{t}, J=10.0 \mathrm{~Hz}, 3 \mathrm{H}), 1.12(\mathrm{t}, J=10.0 \mathrm{~Hz}, 3 \mathrm{H}) ;{ }^{13} \mathrm{C} \mathrm{NMR}$ $\left(125 \mathrm{MHz} \mathrm{CDCl}_{3}\right) \delta 188.7,162.4,159.2,154.9,153.3,147.5$, 146.2 , 137.8, 137.5, 137.0, 131.6, 129.6, 129.1, 128.6, 128.1, $127.6,127.4,127.2,126.4,126.3,116.8,112.5,108.5,85.2,62.7$, $60.9,60.7,58.3,44.0,42.3,14.1,14.0$.

Diethyl 2'-benzyl-2-(4-methoxyphenyl)-3-(2-oxo-2-( $p$-tolyl) ethyl)-2,3-dihydro-2' $H$-spiro[indene-1,3'-isoxazole]-4' ${ }^{\prime}, 5^{\prime}$-dicar-

boxylate (5laa), $29.0: 1 \mathrm{dr}$. Purified by silica gel column chromatography (petroleum ether/ethyl acetate from $50: 1$ to $10: 1$ ): $222.8 \mathrm{mg}$, 69\% yield; light yellow solid, mp 85-86 ${ }^{\circ} \mathrm{C}$, IR (film) 1738, 1712, 1496, 1393, 1305, $1140 \mathrm{~cm}^{-1}$; ${ }^{1} \mathrm{H}$ NMR (500 MHz, $\left.\mathrm{CDCl}_{3}\right) \delta 7.71(\mathrm{~d}, J=5.0 \mathrm{~Hz}, 2 \mathrm{H}), 7.59(\mathrm{~d}, J=10.0 \mathrm{~Hz}, 2 \mathrm{H}), 7.44-$ $7.39(\mathrm{~m}, 2 \mathrm{H}), 7.36-7.32(\mathrm{~m}, 4 \mathrm{H}), 7.30-7.27$ (m, 3H), 7.17 (d, $J=$ $10.0 \mathrm{~Hz}, 2 \mathrm{H}), 6.78$ (d, $J=10.0 \mathrm{~Hz}, 2 \mathrm{H}), 4.21-4.16(\mathrm{~m}, 2 \mathrm{H}), 4.15-$ $4.05(\mathrm{~m}, 4 \mathrm{H}), 4.01(\mathrm{~d}, J=15.0 \mathrm{~Hz}, 1 \mathrm{H}), 3.75(\mathrm{~s}, 3 \mathrm{H}), 3.52-3.48(\mathrm{~m}$, $2 \mathrm{H}), 3.44-3.38(\mathrm{~m}, 1 \mathrm{H}), 2.37(\mathrm{~s}, 2.9 \mathrm{H}), 1.25(\mathrm{t}, J=10.0 \mathrm{~Hz}, 3.1 \mathrm{H})$, $1.13(\mathrm{t}, J=10.0 \mathrm{~Hz}, 2.9 \mathrm{H}) ;{ }^{13} \mathrm{C} \mathrm{NMR}\left(125 \mathrm{MHz}, \mathrm{CDCl}_{3}\right) \delta 198.9$, $162.5,159.2$, 158.9, 154.6, 148.1, 143.9, 137.7, 137.5, 135.1, 132.7 , 129.6, 129.3, 129.2, 129.0, 128.6, 128.3, 127.6, 127.1, 126.6, 126.3, 113.6, 108.9, 85.3, 62.6, 60.9, 60.6, 57.7, 55.3, 44.1, 42.5, 21.8, 14.1, 14.0 .

Diethyl 2'-benzyl-5-chloro-3-(2-oxo-2-( $p$-tolyl)ethyl)-2-phenyl2,3-dihydro-2' $H$-spiro[indene-1,3'-isoxazole]-4' ${ }^{\prime} 5^{\prime}$-dicarboxylate (5oaa), 12.0 : $1 \mathrm{dr}$. Purified by silica gel column chromatography (petroleum ether/ethyl acetate from $50: 1$ to $10: 1$ ): $211.3 \mathrm{mg}$, $65 \%$ yield; light yellow solid, mp $118-120{ }^{\circ} \mathrm{C}$, IR (film) 1740, 1710, 1454, 1394, 1304, $1143 \mathrm{~cm}^{-1} ;{ }^{1} \mathrm{H}$ NMR (400 MHz, $\mathrm{CDCl}_{3}$ ) $\delta 7.83(\mathrm{~d}, J=8.0 \mathrm{~Hz}, 0.2 \mathrm{H}), 7.71(\mathrm{~d}, J=8.0 \mathrm{~Hz}, 2 \mathrm{H}), 7.65-7.62(\mathrm{~m}$, $2 \mathrm{H}), 7.48(\mathrm{~d}, J=4.0 \mathrm{~Hz}, 1 \mathrm{H}), 7.38-7.16(\mathrm{~m}, 12 \mathrm{H}), 4.21-4.09(\mathrm{~m}$, $6 \mathrm{H}), 3.99(\mathrm{~d}, J=12.0 \mathrm{~Hz}, 1 \mathrm{H}), 3.52-3.38(\mathrm{~m}, 3 \mathrm{H}), 2.40(\mathrm{~s}, 0.2 \mathrm{H})$, $2.38(\mathrm{~s}, 2.7 \mathrm{H}), 1.23(\mathrm{t}, J=8.0 \mathrm{~Hz}, 3 \mathrm{H}), 1.16(\mathrm{t}, J=8.0 \mathrm{~Hz}, 3 \mathrm{H}) ;{ }^{13} \mathrm{C}$ NMR (100 MHz, $\left.\mathrm{CDCl}_{3}\right) \delta 198.3,162.3,159.1,155.1,149.9,144.1$, $137.1,136.7,136.5,135.6,134.8,131.5,129.4,129.0,128.6$, 128.3, 128.2, 127.7, 127.6, 127.5, 127.3, 127.1, 108.1, 84.7, 62.7, 61.0, 60.6, 58.5, 43.6, 42.0, 21.8, 14.1, 14.0.

Diethyl 2'-benzyl-6-methyl-3-(2-oxo-2-( $p$-tolyl)ethyl)-2-phenyl2,3-dihydrospiro[indene-1,3'-isoxazolidine]-4' $5^{\prime}$-dicarboxylate (5raa), $14.8: 1 \mathrm{dr}$. Purified by silica gel column chromatography (petroleum ether/ethyl acetate from $50: 1$ to $10: 1$ ): $198.4 \mathrm{mg}$, $63 \%$ yield; yellowish white solid, mp $145-147^{\circ} \mathrm{C}$, IR (film) 1749 , 1712, 1453, 1305, 1200, $1178 \mathrm{~cm}^{-1}$; ${ }^{1} \mathrm{H}$ NMR (400 MHz, $\mathrm{CDCl}_{3}$ ) $\delta$ 7.70-7.65 (m, 4H), $7.34(\mathrm{t}, J=4.0 \mathrm{~Hz}, 4 \mathrm{H}), 7.30-7.20(\mathrm{~m}, 6 \mathrm{H})$, 
$7.16(\mathrm{~d}, J=8.0 \mathrm{~Hz}, 2 \mathrm{H}), 7.19(\mathrm{~d}, J=8.0 \mathrm{~Hz}, 1 \mathrm{H}), 4.21-4.05(\mathrm{~m}$, $6 \mathrm{H}), 4.00(\mathrm{~d}, J=16.0 \mathrm{~Hz}, 1 \mathrm{H}), 3.53(\mathrm{~d}, J=12.0 \mathrm{~Hz}, 1 \mathrm{H}), 3.49-3.37$ $(\mathrm{m}, 2 \mathrm{H}), 2.37$ (s, 3H), $2.36(\mathrm{~s}, 3 \mathrm{H}), 1.25(\mathrm{t}, J=8.0 \mathrm{~Hz}, 3.2 \mathrm{H}), 1.13$ $(\mathrm{t}, J=8.0 \mathrm{~Hz}, 2.8 \mathrm{H}) ;{ }^{13} \mathrm{C} \mathrm{NMR}\left(100 \mathrm{MHz}, \mathrm{CDCl}_{3}\right) \delta 199.0,162.5$, 159.2 , 154.5, 145.0, 143.8, 137.8, 137.5, 137.3, 136.8, 135.1, $131.6,130.6$, 129.3, 129.1, 128.6, 128.3, 128.2, 127.6, 127.3, 126.6, 126.2 , 123.0, 109.0, 100.1, 93.3, 85.3, 62.6, 60.8, 60.6, 58.6, 43.7, 42.6, 21.8, 21.6, 14.1, 14.0.

Dimethyl 2'-benzyl-3-(2-oxo-2-( $p$-tolyl)ethyl)-2-phenyl-2,3dihydro-2' $H$-spiro[indene-1,3'-isoxazole]- $4^{\prime}, 5^{\prime}$-dicarboxylate

(5dab), 11.0 : 1 dr. Purified by silica gel column chromatography (petroleum ether/ethyl acetate from $50: 1$ to $10: 1$ ): $241.0 \mathrm{mg}, 82 \%$ yield; light yellow solid, $\mathrm{mp} 132-134{ }^{\circ} \mathrm{C}$, IR (film) 1765, 1750, 1448, 1352, 1307, $1140 \mathrm{~cm}^{-1} ;{ }^{1} \mathrm{H}$ NMR $(400 \mathrm{MHz}$, $\left.\mathrm{CDCl}_{3}\right) \delta 7.68(\mathrm{t}, J=8.0 \mathrm{~Hz}, 4 \mathrm{H}), 7.47-7.41(\mathrm{~m}, 2 \mathrm{H}), 7.36-7.20(\mathrm{~m}$, $10 \mathrm{H}), 7.16(\mathrm{~d}, J=8.0 \mathrm{~Hz}, 2 \mathrm{H}), 4.19-4.10(\mathrm{~m}, 2 \mathrm{H}), 4.00(\mathrm{~d}, J=$ $16.0 \mathrm{~Hz}, 1 \mathrm{H}), 3.73(\mathrm{~s}, 3 \mathrm{H}), 3.66(\mathrm{~s}, 3 \mathrm{H}), 3.52-3.39(\mathrm{~m}, 3 \mathrm{H}), 2.39$ (s, $0.3 \mathrm{H}), 2.37(\mathrm{~s}, 2.7 \mathrm{H}) ;{ }^{13} \mathrm{C} \mathrm{NMR}\left(100 \mathrm{MHz}, \mathrm{CDCl}_{3}\right) \delta$ 198.8, 162.9, 159.5 , 154.4, 148.0, 143.9, 137.6, 137.3, 137.1, 135.0, 131.6, $129.7,129.3,128.9,128.6,128.3,128.2,127.6,127.4,127.3$, 126.7, 126.1, 109.2, 85.3, 60.7, 58.4, 53.3, 52.1, 44.0, 42.4, 21.8.

Diethyl 2'-methyl-3-(2-oxo-2-( $p$-tolyl)ethyl)-2-phenyl-2,3-dihydro-spiro[indene-1,3'-isoxazolidine]- $4^{\prime}, 5^{\prime}$-dicarboxylate (5dca), $7.3: 1$ dr. Purified by silica gel column chromatography (petroleum ether/ethyl acetate from $50: 1$ to $10: 1$ ): $121.4 \mathrm{mg}$, $45 \%$ yield; brown solid, $\mathrm{mp} 74-76^{\circ} \mathrm{C}$, IR (film) 1737, 1702, 1460, 1370, 1291, $1186 \mathrm{~cm}^{-1}$; ${ }^{1} \mathrm{H}$ NMR (500 MHz, $\left.\mathrm{CDCl}_{3}\right) \delta 7.84(\mathrm{~d}, J=$ $10.0 \mathrm{~Hz}, 0.3 \mathrm{H}), 7.72(\mathrm{~d}, J=10.0 \mathrm{~Hz}, 1.7 \mathrm{H}), 7.68(\mathrm{~d}, J=5.0 \mathrm{~Hz}$, $1.7 \mathrm{H}), 7.44(\mathrm{~d}, J=5.0 \mathrm{~Hz}, 0.3 \mathrm{H}), 7.37(\mathrm{~d}, J=10.0 \mathrm{~Hz}, 1 \mathrm{H}), 7.32(\mathrm{t}$, $J=5.0 \mathrm{~Hz}, 1 \mathrm{H}), 7.29-7.19(\mathrm{~m}, 7 \mathrm{H}), 7.14(\mathrm{~d}, J=10.0 \mathrm{~Hz}, 0.2 \mathrm{H})$, 4.26-4.14 (m, 2.3H), 4.13-4.04 (m, 3.5H), 3.43 (dd, $J=15.0$, $5.0 \mathrm{~Hz}, 0.9 \mathrm{H}), 3.32(\mathrm{dd}, J=15.0,10.0 \mathrm{~Hz}, 1 \mathrm{H}), 2.62(\mathrm{~s}, 2.6 \mathrm{H}), 2.45$ $(\mathrm{s}, 0.4 \mathrm{H}), 2.40(\mathrm{~s}, 0.4 \mathrm{H}), 2.38(\mathrm{~s}, 2.6 \mathrm{H}), 1.31-1.19(\mathrm{~m}, 3.4 \mathrm{H}), 1.11$ $(\mathrm{t}, J=10.0 \mathrm{~Hz}, 2.6 \mathrm{H}) ;{ }^{13} \mathrm{C} \mathrm{NMR}\left(125 \mathrm{MHz}, \mathrm{CDCl}_{3}\right) \delta 199.0,162.6$, $159.1,153.8,148.0,143.8,137.3,137.2$, 135.1, 131.8, 130.4, $129.9,129.5,129.4,129.4,128.5,128.3,128.0,127.9,127.4$, 126.9, 126.5, 126.5, 108.9, 85.6, 62.6, 60.9, 58.1, 43.9, 43.7, 42.5, 21.8, 14.1, 14.0. HRMS (ESI) calcd for $\mathrm{C}_{33} \mathrm{H}_{34} \mathrm{NO}_{6}[\mathrm{M}+\mathrm{H}]^{+}$ 540.2381 , found 540.2386 .

Procedure for the synthesis of allylic alcohol 6 from spiroindenyl isoxazoline 5daa (Scheme 2). To a dried Schlenk flask was charged with 5 daa $(0.1847 \mathrm{~g}, 0.30 \mathrm{mmol})$, zinc dust $(0.1962 \mathrm{~g}, 3.00 \mathrm{mmol}), \mathrm{NH}_{4} \mathrm{Cl}(0.3210 \mathrm{~g}, 6.00 \mathrm{mmol})$, and $\mathrm{MeOH}$ $(3.0 \mathrm{~mL})$ under air atmosphere. The reaction mixture was stirred at $75{ }^{\circ} \mathrm{C}$ for $5 \mathrm{~h}$, then filtered through a short pad of silica gel and washed with ethyl acetate. After removal of solvent, the crude residue was purified by flash chromatography on silica gel (petroleum ether/ethyl acetate $=5: 1, \mathrm{v} / \mathrm{v}$ ) to afford the product 6 as a white solid ( $0.1153 \mathrm{~g}, 75 \%$ yield $), \mathrm{mp} .50-52{ }^{\circ} \mathrm{C}$, IR (film) 3486, 1730, 1680, 1606, 1453, 1366, 1224, $1079 \mathrm{~cm}^{-1} ;{ }^{1} \mathrm{H}$ NMR (500 MHz, $\left.\mathrm{CDCl}_{3}\right) \delta 8.02(\mathrm{~d}, J=10.0 \mathrm{~Hz}, 0.1 \mathrm{H}), 7.88-7.82$ $(\mathrm{m}, 0.3 \mathrm{H}), 7.75(\mathrm{~d}, J=10.0 \mathrm{~Hz}, 0.1 \mathrm{H}), 7.67(\mathrm{~d}, J=5.0 \mathrm{~Hz}, 0.7 \mathrm{H})$, $7.57-7.51(\mathrm{~m}, 1.9 \mathrm{H}), 7.42(\mathrm{~d}, J=10.0 \mathrm{~Hz}, 0.2 \mathrm{H}), 7.40-7.35(\mathrm{~m}$, $1 \mathrm{H}), 7.32-7.22(\mathrm{~m}, 2.6 \mathrm{H}), 7.19(\mathrm{~d}, J=5.0 \mathrm{~Hz}, 0.3 \mathrm{H}), 7.17-7.13(\mathrm{~m}$, $2 \mathrm{H}), 7.04-6.96(\mathrm{~m}, 2.8 \mathrm{H}), 6.85(\mathrm{~d}, J=5.0 \mathrm{~Hz}, 0.2 \mathrm{H}), 6.74(\mathrm{t}, J=$ $10.0 \mathrm{~Hz}, 1.7 \mathrm{H}), 5.79(\mathrm{~d}, J=10.0 \mathrm{~Hz}, 0.2 \mathrm{H}), 5.47$ (d, $J=10.0 \mathrm{~Hz}$, $0.2 \mathrm{H}), 5.05-5.02(\mathrm{~m}, 0.8 \mathrm{H}), 4.87(\mathrm{~d}, J=10.0 \mathrm{~Hz}, 0.8 \mathrm{H}), 3.40(\mathrm{t}, J=$
$10.0 \mathrm{~Hz}, 0.9 \mathrm{H}), 4.31-4.24(\mathrm{~m}, 2.1 \mathrm{H}), 4.10-3.90(\mathrm{~m}, 0.5 \mathrm{H}), 3.89-$ $3.75(\mathrm{~m}, 1.8 \mathrm{H}), 3.43(\mathrm{~d}, J=5.0 \mathrm{~Hz}, 0.8 \mathrm{H}), 3.19-3.01(\mathrm{~m}, 1 \mathrm{H})$, 2.84-2.74 (m, 0.9H), $2.41(\mathrm{~s}, 0.2 \mathrm{H}), 2.37(\mathrm{~s}, 0.5 \mathrm{H}), 2.36(\mathrm{~s}, 2.2 \mathrm{H})$, $1.30-1.21(\mathrm{~m}, 3.5 \mathrm{H}), 1.11(\mathrm{t}, J=5.0 \mathrm{~Hz}, 0.5 \mathrm{H}), 0.82(\mathrm{t}, J=10.0 \mathrm{~Hz}$, $2.1 \mathrm{H}) ;{ }^{13} \mathrm{C}$ NMR $\left(125 \mathrm{MHz}, \mathrm{CDCl}_{3}\right) \delta 198.9,173.4,173.3,167.5$, $156.5,152.7,150.0,143.8,140.6,140.3,139.2,134.7,131.0$, $130.5,129.5,129.3,129.2,128.9,128.5,128.5,128.2,128.1$, $128.0,127.5$, 127.0, 127.0, 126.9, 126.7, 126.4, 125.7, 124.9, 124.6, 124.0, 70.3, 68.6, 62.3, 62.0, 61.2, 60.9, 55.4, 54.8, 43.4, 43.1, 39.7, 39.0, 21.8, 14.3, 14.2, 14.0, 13.8; HRMS (ESI) calcd for $\mathrm{C}_{32} \mathrm{H}_{33} \mathrm{O}_{6}[\mathrm{M}+\mathrm{H}]^{+}$513.2272, found 513.2277.

Procedure for the $\mathrm{Co}_{2}(\mathrm{CO})_{8}$ catalyzed rearrangement of spiroindenyl isoxazoline $5 \mathrm{raa}$ (Scheme 2). To a dried Schlenk flask was charged with 5 raa $(0.1260 \mathrm{~g}, 0.20 \mathrm{mmol}), \mathrm{Co}_{2}(\mathrm{CO})_{8}$ $(0.0342 \mathrm{~g}, 0.10 \mathrm{mmol})$, and $\mathrm{MeCN}(4.0 \mathrm{~mL})$ under argon. The reaction mixture was stirred at $100{ }^{\circ} \mathrm{C}$ for $12 \mathrm{~h}$, then filtered through a short pad of silica gel and washed with ethyl acetate. After removal of solvent, the crude residue was purified by flash chromatography on silica gel (petroleum ether/ethyl acetate = $5: 1, \mathrm{v} / \mathrm{v})$ to afford the product 7 as a light yellow solid $(0.0655 \mathrm{~g}$, $52 \%$ yield), mp $45-47^{\circ} \mathrm{C}$, IR (film) 1733, 1710, 1679, 1494, 1284, 1215, $1093 \mathrm{~cm}^{-1} ;{ }^{1} \mathrm{H}$ NMR (400 $\left.\mathrm{MHz}, \mathrm{CDCl}_{3}\right) \delta 7.89(\mathrm{~d}, J=$ $8.0 \mathrm{~Hz}, 0.3 \mathrm{H}), 7.77(\mathrm{~d}, J=8.0 \mathrm{~Hz}, 1.7 \mathrm{H}), 7.39-7.33(\mathrm{~m}, 2 \mathrm{H}), 7.29$ $(\mathrm{d}, J=8.0 \mathrm{~Hz}, 2 \mathrm{H}), 7.15-7.09(\mathrm{~m}, 4 \mathrm{H}), 7.07-6.98(\mathrm{~m}, 3 \mathrm{H}), 5.48(\mathrm{~d}$, $J=16.0 \mathrm{~Hz}, 0.8 \mathrm{H}), 5.20(\mathrm{~d}, J=16.0 \mathrm{~Hz}, 0.2 \mathrm{H}), 4.69(\mathrm{~d}, J=$ $16.0 \mathrm{~Hz}, 0.2 \mathrm{H}), 4.19$ (d, $J=16.0 \mathrm{~Hz}, 0.9 \mathrm{H}), 4.08-4.02(\mathrm{~m}, 1.3 \mathrm{H})$, 3.98-3.92 (m, 1.7H), 3.83-3.73 (m, 2.9H), 3.62-3.56 (m, 0.2H), $3.32-3.26(\mathrm{~m}, 0.9 \mathrm{H}), 3.15-3.03(\mathrm{~m}, 0.3 \mathrm{H}), 2.89$ (dd, $J=16.0$, $4.0 \mathrm{~Hz}, 0.9 \mathrm{H}), 2.44(\mathrm{~s}, 2.5 \mathrm{H}), 2.38(\mathrm{~s}, 0.5 \mathrm{H}), 2.36(\mathrm{~s}, 2.5 \mathrm{H}), 2.08(\mathrm{~s}$, $0.5 \mathrm{H}), 1.07$ (d, $J=8.0 \mathrm{~Hz}, 0.6 \mathrm{H}), 1.03-0.97(\mathrm{~m}, 5.4 \mathrm{H}) ;{ }^{13} \mathrm{C} \mathrm{NMR}$ $\left(100 \mathrm{MHz}, \mathrm{CDCl}_{3}\right) \delta 197.9,165.8,162.0,161.7,144.3,141.5$, 141.4 , 137.3, 136.8, 135.7, 134.8, 131.9, 131.8, 130.9, 130.6, $129.5,129.4,129.3,129.2,128.6,128.5,128.5,128.5,128.4$, $128.4,128.3,127.8,127.7,127.3,127.0,62.3,61.7,61.4,51.4$, 46.0, 45.9, 45.4, 44.9, 44.0, 40.2, 21.9, 21.6, 13.8, 13.7; HRMS (ESI) calcd for $\mathrm{C}_{40} \mathrm{H}_{40} \mathrm{NO}_{6}[\mathrm{M}+\mathrm{H}]^{+}$630.2850, found 630.2857.

\section{Conflicts of interest}

There are no conflicts to declare.

\section{Acknowledgements}

We thank the National Natural Science Foundation of China (No. 21502065, 21702072), Scientific Research Fund of Hunan Provincial Education Department (No. 19A389), Opening Fund of CAS Key Laboratory of Molecular Recognition and Function (Chinese Academy of Sciences) (No. 2017LRMF009) for financial support.

\section{Notes and references}

1 (a) A. G. Habeeb, P. N. Praveen Rao and E. E. Knaus, J. Med. Chem., 2001, 44, 2921-2927; (b) R. D. Cramer, R. J. Jilek, S. Guessregen, S. J. Clark, B. Wendt and R. D. Clark, J. Med. Chem., 2004, 47, 6777-6791; (c) A. I. Hubich, T. A. Zheldakova, T. V. Chernikhova, E. V. Koroleva, 
F. A. Lakhvich and M. V. Sholukh, Biochem. Biophys. Res. Commun., 2006, 341, 357-362.

2 For selected examples: (a) A. Brandi, F. Cardona, S. Cicchi, F. M. Cordero and A. Goti, Chem.-Eur. J., 2009, 15, 78087821; (b) H. Wei, C. Qiao, G. Liu, Z. Yang and C. Li, Angew. Chem., Int. Ed., 2013, 52, 620-624; and references therein.

3 Selected reviews: (a) J. P. Freeman, Chem. Rev., 1983, 83, 241261; (b) T. M. V. D. Pinho e Melo, Eur. J. Org. Chem., 2010, 3363-3376; (c) N. V. Chukanov and V. A. Reznikov, Russ. Chem. Bull., Int. Ed., 2011, 60, 379-399.

4 (a) I. S. Young and M. A. Kerr, Org. Lett., 2004, 6, 139-141; (b) P.-W. Xu, C. Chen, J.-K. Liu, Y.-T. Song, F. Zhou, J. Yan and J. Zhou, J. Org. Chem., 2018, 83, 12763-12774.

5 (a) S. A. Hussain, A. H. Sharma and M. J. Perkin, J. Chem. Soc. D, 1979, 289-291; (b) K. M. Partridge, M. E. Anzovino and T. P. Yoon, J. Am. Chem. Soc., 2008, 130, 2920-2921; (c) A. R. Reddy, Z. Guo, F.-M. Siu, C.-N. Lok, F. Liu, K.-C. Yeung, C.-Y. Zhou and C.-M. Che, Org. Biomol. Chem., 2012, 10, 9165-9174; (d) H. Hou, S. Zhu, F. Pan and M. Rueping, Org. Lett., 2014, 16, 2872-2875; (e) Y. Liu, J. Ao, S. Paladhi, C. E. Song and H. Yan, J. Am. Chem. Soc., 2016, 138, 16486-16492; (f) C. Gioia, F. Fini, A. Mazzanti, L. Bernardi and A. Ricci, J. Am. Chem. Soc., 2009, 131, 9614-9615; (g) Z.-J. Xu, D. Zhu, X. Zeng, F. Wang, B. Tan, Y. Hou, Y. Lv and G. Zhong, Chem. Commun., 2010, 46, 2504-2506; (h) N. Morita, R. Kono, K. Fukui, A. Miyazawa, H. Masu, I. Azumaya, S. Ban, Y. Hashimoto, I. Okamoto and O. Tamura, J. Org. Chem., 2015, 80, 4797-4802; (i) X. Li, T. Feng, D. Li, H. Chang, W. Gao and W. Wei, J. Org. Chem., 2019, 84, 4402-4412.

6 (a) X. Guinchard, Y. Vallée and J.-N. Denis, Org. Lett., 2005, 7, 5147-5150; (b) A. Gini, M. Segler, D. Kellner and O. G. Mancheño, Chem.-Eur. J., 2015, 21, 12053-12060; (c) A. R. Reddy, C.-Y. Zhou and C.-M. Che, Org. Lett., 2014, 16, 1048-1051; (d) K. Wu, C.-Y. Zhou and C.-M. Che, Org. Lett., 2019, 21, 85-89; (e) X. Li, T. Feng, D. Li, H. Chang, W. Gao and W. Wei, J. Org. Chem., 2020, 85, 9538-9547.

7 D. P. Canterbury, Il. R. Herrick, J. Um, K. N. Houk and A. J. Frontier, Tetrahedron, 2009, 65, 3165-3179.

8 G. S. Jang, J. Lee, J. Seo and S. K. Woo, Org. Lett., 2017, 19, 6448-6451.

9 Y. Zheng, C. M. Tice and S. B. Singh, Bioorg. Med. Chem. Lett., 2014, 24, 3673-3682.

10 (a) J. Y. Pfeiffer and A. M. Beauchemin, J. Org. Chem., 2009, 74, 8381-8383; (b) S. Franco, F. L. Merchán, P. Merino and T. Tejero, Synth. Commun., 1995, 25, 2275-2284.

11 (a) C.-K. Pei, Yu. Jiang and M. Shi, Eur. J. Org. Chem., 2012, 4206-4216; (b) M. Mehrdad, L. Faraji and K. Jadidi, Monatsh. Chem., 2011, 142, 917-921; (c) J. Feng, P.-J. Ma, Y.-M. Zeng, Y.-J. Xu and C.-D. Lu, Chem. Commun., 2018, 54, 2882-2885; (d) L. Maiuolo, P. Merino, V. Algieri, M. Nardi, M. L. D. Gioia, B. Russo, I. Delso, M. A. Tallarida and A. D. Nino, RSC Adv., 2017, 7, 48980-48988; (e) C.-H. Chen, Q.-Q. Liu, X.-P. Ma, Y. Feng, C. Liang, C.-X. Pan, G.-F. Su and D.-L. Mo, J. Org. Chem., 2017, 82, 6417-6425; $(f)$ H.-B. Yang and M. Shi, Org. Biomol. Chem., 2012, 10, 8236-8243.

12 For selected examples, see: (a) J. Rong, P. Roselt, J. Plavec and J. Chattopadhyaya, Tetrahedron, 1994, 50, 4921-4936; (b) S. Torrente, B. Noya, M. D. Paredes and A. Alonso, J. Org. Chem., 1997, 62, 6710-6711; (c) T. S. orrente, B. Noya, V. Branchadell and R. Alonso, J. Org. Chem., 2003, 68, 4772-4783.

13 (a) A. W. Johnson, J. Org. Chem., 1963, 28, 252-254; (b) M. A. Abou-Gharbia and M. M. Joullie, J. Org. Chem., 1979, 44, 2961-2966; (c) D.-L. Mo, D. A. Wink and L. L. Anderson, Org. Lett., 2012, 14, 5180-5183; (d) W. H. Pecak, J. Son, A. J. Burnstine and L. L. Anderson, Org. Lett., 2014, 16, 3440-3443.

14 M. Cordier and A. Archambeau, Org. Lett., 2018, 20, 22652268.

15 Y. Luo, C.-H. Chen, J.-Q. Zhang, C. Liang and D.-L. Mo, Synthesis, 2020, 52, 424-432.

16 (a) B. B. Snider and H. Lin, J. Am. Chem. Soc., 1999, 121, 7778-7786; (b) B. B. Snider and H. Lin, Org. Lett., 2000, 2, 643-646; (c) K. Shuji and T. Takashi, Chem. Lett., 1995, 24, 49-50.

17 (a) R. Natarajan, P. A. Unnikrishnan, S. Radhamani, J. P. Rappai and S. Prathapan, Tetrahedron Lett., 2016, 57(27-28), 2981-2984; (b) S. Radhamani, R. Natarajan, P. A. Unnikrishnan, S. Prathapan and J. P. Rappai, New J. Chem., 2015, 39, 5580-5588.

18 For selected examples about detailed calculation on the mechanism of $[3+2]$ cycloaddition reaction, please see: $(a)$ R. Jasiński, M. Ziółkowska, O. M. Demchuk and A. Maziarka, Cent. Eur. J. Chem., 2014, 12, 586-593; (b) R. Jasiński, RSC Adv., 2015, 5, 101045-101048; (c) R. Jasiński, Tetrahedron Lett., 2015, 56, 532-535; (d) R. Jasiński, Comput. Theor. Chem., 2018, 1125, 77-85; (e) R. Jasiński, J. Mol. Graphics Modell., 2020, 94, 107461107465; (f) R. Jasiński and E. Dresler, Organics, 2020, 1, 4969.

19 R. Jasiński, K. Mróz and A. Kącka, J. Heterocycl. Chem., 2016, 53, 1424-1429.

20 Y. Liu, X. Feng, Y. Liu, H. Lin, Y. Li, Y. Gong, L. Cao and L. Chen, Org. Lett., 2019, 21, 382-386.

21 (a) F. A. Khan, J. Dash, C. Sudheer and R. K. Gupta, Tetrahedron Lett., 2003, 44, 7783-7787; (b) S. M. Kelly and B. H. Lipshutz, Org. Lett., 2014, 16, 98-101.

22 T. Ishikawa, T. Kudoh, J. Yoshida, A. Yasuhara, S. Manabe and S. Saito, Org. Lett., 2002, 4, 1907-1910.

23 B. Prüger, G. E. Hofmeister, C. B. Jacobsen, D. G. Alberg, M. Nielsen and K. A. Jørgensen, Chem.-Eur. J., 2010, 16, 3783-3790. 